\title{
TRAVELING VORTEX HELICES FOR SCHRÖDINGER MAP EQUATIONS
}

\author{
JUNCHENG WEI AND JUN YANG
}

\begin{abstract}
We construct traveling wave solutions with vortex helix structures for the Schrödinger map equation

$$
\frac{\partial m}{\partial t}=m \times\left(\Delta m-m_{3} \vec{e}_{3}\right) \quad \text { on } \mathbb{R}^{3} \times \mathbb{R},
$$

of the form $m\left(s_{1}, s_{2}, s_{3}-\delta|\log \epsilon| \epsilon t\right)$ with traveling velocity $\delta|\log \epsilon| \epsilon$ along the direction of $s_{3}$ axis. We use a perturbation approach which gives a complete characterization of the asymptotic behavior of the solutions.
\end{abstract}

\section{INTRODUCTION}

The aim of this paper is to construct traveling wave solutions for a class of Landau-Lifshitz equations. We shall concentrate on the traveling wave solutions of the Schrödinger map equation

$$
\frac{\partial m}{\partial t}=m \times\left(\Delta m-m_{3} \vec{e}_{3}\right) \quad \text { in } \mathbb{R}^{N} \times \mathbb{R},
$$

or equivalently the equation

$$
-m \times \frac{\partial m}{\partial t}=\Delta m-m_{3} \vec{e}_{3}+\left(|\nabla m|^{2}+m_{3}^{2}\right) m .
$$

Here $m: \mathbb{R}^{N} \times \mathbb{R} \rightarrow \mathbb{S}^{2}$ so that $|m(s, t)| \equiv 1$ and where $\vec{e}_{3}=(0,0,1) \in \mathbb{R}^{3}$.

The equation (1.1) (or equivalently (1.2)) is, in fact, the Landau-Lifshitz equation describing the planar ferromagnets, that is, ferromagnets with an easy-plane anisotropy ([32], [38]). The unit normal to the easy-plane is assumed to be $\vec{e}_{3}$ in the equations, see for example [38]. Despite some serious efforts (see e.g. [15], [16], [43], [44], [21], [9, 24, 39], [22, 23], [19, 20, 17, 18]) and the references therein), some basic mathematical issues such as local and global well-posedness and global in time asymptotics for the equation (1.1) remain unknown. If one is interested in one-dimensional wave (plane-wave) solutions of (1.1), that is, $m: \mathbb{R} \times \mathbb{R} \rightarrow \mathbb{S}^{2}$ (or $\mathbb{S}^{1}$ ), a lot were known as (1.1) becomes basically an integrable system (see [44] and [2]). The problem in 2-D or higher dimensions are much more subtle. Even though it is possible to obtain weak solutions of (1.1) (see [2], [36], [42], [43]-[44] ), one does not know if such weak solutions are classical (smooth) or unique.

From the physical side of view, one expects topological solitons, which are half magnetic bubbles, exist in solutions of (1.1) (see [27] and [38]). Indeed, in [25]-[26], F. Hang and F. Lin have established the corresponding static theory for such magnetic vortices. They are very much like vortices in the superconductor described by the Ginzburg-Landau equation, see [3] and references therein.

Let us recall the essential features of vortex dynamics in a classical fluid (or a superfluid modeled by the Gross-Pitaevskii equation, see [37]). A single vortex or antivortex is always spontaneously pinned and hence can move only together with the background fluid. However vortex motion relative to the fluid is possible in the presence of other vortices and it displays characteristics similar to the 2-D Hall motion of interacting electric charges in a uniform magnetic field. In particular, two like vortices orbit around each other while a vortex-antivortex pair undergoes

2010 Mathematics Subject Classification. 35B06, 35B40, 35J25, 35J20.

Key words and phrases. Traveling Waves, Schrödinger Map equations, Vortex Helices, Perturbations.

Corresponding author: Jun Yang, jyang@szu.edu.cn. 
Kelvin motion along parallel trajectories that are perpendicular to the line connecting the vortex and the antivortex. This latter fact had been obtained in a special case by Jones and Robert [29]. They also asymptotically derived a three dimensional (3-D) solitary wave that describe a vortex ring moving steadily along its symmetric axis. For more precise mathematical proofs, there are some works for the case of Gross-Pitaevskii equation

$$
i \frac{\partial u}{\partial t}=\Delta u+u-|u|^{2} u \quad \text { in } \quad \mathbb{R}^{N} \times \mathbb{R}
$$

In two dimensional plane, F. Bethuel and J. Saut constructed a traveling wave with two vortices of degree \pm 1 in [8]. In higher dimension, by minimizing the energy, F. Bethuel, G. Orlandi and D. Smets constructed solutions with a vortex ring [7], see also [5]. See also [10] for another proof by Mountain Pass Lemma. The reader can refer to the review paper [6] by F. Bethuel, P. Gravejat and J. Saut and the references therein.

By a completely different method, F. Lin and J. Wei [34] obtained the results of existence of solutions, similar as those of [8], [7], for the Landau-Lifshitz equation (1.1). More precisely, if we look for a solution of the traveling wave $m\left(s^{\prime}, s_{N}-C t\right)$ of the equation (1.1), then $m$ must be a solution of

$$
-C \frac{\partial m}{\partial s_{N}}=m \times\left(\Delta m-m_{3} \vec{e}_{3}\right) .
$$

Here, it travels in the $s_{N}$-direction with the speed $C$, which will be set in the form

$$
C=c \epsilon \quad \text { with } \quad c>0 \text { and } \epsilon>0 .
$$

After a proper scaling in the space, (1.4) becomes

or

$$
-c \frac{\partial m}{\partial s_{N}}=m \times\left(\Delta m-\frac{m_{3}}{\epsilon^{2}} \vec{e}_{3}\right), \quad s \in \mathbb{R}^{N},
$$

$$
c m \times \frac{\partial m}{\partial s_{N}}=\Delta m-\frac{m_{3} \vec{e}_{3}}{\epsilon^{2}}+\left(|\nabla m|^{2}+\frac{m_{3}^{2}}{\epsilon^{2}}\right) m .
$$

The main results of the paper [34] are the following

Theorem 1.1. [34] Let $N \geq 2$ and $\epsilon$ sufficiently small there is an axially symmetric solution $m=m\left(\left|s^{\prime}\right|, s_{N}\right) \in C^{\infty}\left(\mathbb{R}^{N}, \mathbb{S}^{2}\right)$ of (1.6) such that

$$
E_{\epsilon}(m)=\int_{\mathbb{R}^{N}} \frac{1}{2}\left(|\nabla m|^{2}+\frac{\left|m_{3}\right|^{2}}{\epsilon^{2}}\right) \mathrm{d} s<\infty
$$

and that $m$ has exactly one vortex at $\left(\left|s^{\prime}\right|, s_{N}\right)=\left(a_{\epsilon}, 0\right)$ of degree +1 , where $a_{\epsilon} \approx \frac{1}{2}$. If $N=2$, the traveling velocity $C \sim \epsilon$, while $C=(N-2) \epsilon\left|\log \frac{1}{\epsilon}\right|$ for $N \geq 3$.

Naturally such solution $m$ gives rise to a nontrivial (two-dimensional) traveling wave solution of (1.1) with a pair of vortex and antivortex which undergoes the Kelvin Motion as described above. Solutions constructed in Theorem 1.1 are called traveling vortex rings for the case of the dimension $N \geq 3$. We note that formal arguments as well as numerical evidences were already presented in the work [38]. We should also note that in the case of the initial date of (1.1) contains only one vortex (one magnetic half-bubble), with its structure as described in the work of [25]-[26] very precisely, the above discussions imply that the vortex will simply stay at its center of mass, and a meaningful mathematical issue to examine would be its global stability. It is, however, unknown to authors that whether such stability result is true or not, see [22], [23], [24] for relevant discussions. On the other hand, it is relatively easy to generalize the work of [35] to the equation (1.1) for the planar ferromagnets. One may obtain the same Kirchhoff vortex dynamical law for these widely separated and slowly moving magnetic half-bubbles, see [33] (as formally derived in [38] and also [37] for the Gross-Pitaevskii equation) of solutions of (1.1).

In the present paper, we concern the existence of a traveling wave solution possessing vortex helix structures for problem (1.1) by construction a symmetric solution to (1.4). In this direction, 
we refer the paper by D. Chiron [11] for the existence of vortex helices for Gross-Pitaevskii equation (1.3). For the Allen-Cahn equation, there are also solutions with phase transition layers invariant under screw-motions [14]. The main result reads

Theorem 1.2. Let $N=3$. For any sufficiently small traveling velocity $C$, there is an axially symmetric solution $m=m\left(\left|s^{\prime}\right|, s_{N}\right) \in C^{\infty}\left(\mathbb{R}^{3}, \mathbb{S}^{2}\right)$ of (1.4). Moreover, by the transformation

$$
u=\frac{m_{1}+i m_{2}}{1+m_{3}} \in \mathbb{C}
$$

$u$ has a vortex helix of degree +1 directed along the curve in the form

$$
\alpha \in \mathbb{R} \mapsto(\hat{d} \cos \alpha, \hat{d} \sin \alpha, \hat{\lambda} \alpha) \in \mathbb{R}^{3},
$$

for any parameters $\hat{d}>0$ and $\hat{\lambda} \neq 0$, and $u$ is also invariant under the screw motion expressed in cylinder coordinates

$$
\left(r, \Psi, s_{3}\right) \mapsto\left(r, \Psi+\alpha, s_{3}+\hat{\lambda} \alpha\right), \quad \forall \alpha \in \mathbb{R}
$$

In other words, we have found a traveling wave solution to (1.1). The traveling velocity is

$$
C=\frac{1}{\sqrt{\hat{d}^{2}+\hat{\lambda}^{2}}} \epsilon \log \frac{1}{\epsilon} .
$$

Some words of discussions are in order to end the introduction. The traveling velocity in (1.9) depends on the geometric parameters of the helix (cf. (1.5), (3.1) and (3.10)). To get a nontrivial vortex helix, we assume that the parameters $\hat{d}>0$ and $\hat{\lambda} \neq 0$. Note that $\hat{\lambda}=0$ will go back to the case of vortex ring in Theorem 1.1. We can choose $\epsilon$ so that $C$ in (1.9) is small enough in such a way that we can use the reduction method. For more precise asymptotic behaviors of the solutions, the reader can refer to the details in Sections 3-4.

A natural problem is to construct higher dimensional vortex phenomena with invariance under skew motions in Euclidean spaces. According to [12], there are helix submanifolds in any Euclidean space of odd dimension. We can use the same method to show the existence of vortex phenomena on some special helix submanifolds. The reader can refer to Remark 3.1.

Another interesting question is the existence of a solution with a double-helix vortex structure. Helices are vital for life as we know it, the DNA polymer being a famous example of a double-helix structure [41]. In fluid studies, theory on helical vortices assumes possible existence of an arbitrary number of interacting helical vortex filaments, especially the existence of double-helix structures [1]. It is supposed that spiral pairing is the elementary interaction in turbulence. A double-helix structure formed by a pair of vortices was also studied by the numerical method for the helical spin textures in ferromagnetic spin-1 Bose-Einstein condensates subject to dipolar inter-particle forces [28].

Here is the outline of the approach. We use the standard reduction method (cf. [13], [34]) to construct the solutions with vortex helices to problem (1.4) with dimension $N=3$. By using the screw invariance of solutions, we first transform the problem to a two-dimensional case (3.12) with boundary condition (3.14) on the infinite strip $\mathfrak{S}$ (cf. (3.13)). Note that problem (3.12) is degenerate when $x_{1}=0$ due to the terms

$$
\frac{1}{x_{1}} \frac{\partial u}{\partial x_{1}} \text { and } \gamma^{-2}\left(1+\frac{\lambda^{2}}{x_{1}^{2}}\right) \frac{\partial^{2} u}{\partial x_{2}^{2}} .
$$

The second degenerate term in the above did not appear in the arguments of the works [7], [10] and [34] for the existence of traveling vortex rings. Note that the paper [11] claimed the existence of solution with helix structures and screw motion invariance in Theorem 3 without a proof. So there was no need to handle the second degenerate term therein. The reader can refer to Theorem 3 and its remark in [11]. Here, we shall make careful analysis to deal with the problem near the 
origin. After that, the key point is then to construct a solution with a vortex of degree +1 at $(d, 0)$ and its antipair of degree -1 at $(-d, 0)$. Additional to the computations for standard vortices in two dimensional case, there are two extra derivative terms

$$
\frac{1}{x_{1}} \frac{\partial u}{\partial x_{1}} \quad \text { and } \quad \gamma^{-2}\left(\frac{\lambda^{2}}{x_{1}^{2}}-\frac{\lambda^{2}}{d^{2}}\right) \frac{\partial^{2} u}{\partial x_{2}^{2}}
$$

If we put $w=\rho(\ell) e^{i \theta}$ as the approximate solution to (3.12) in the vortex core region, then there exist two singular terms (cf. (4.16) and (4.11)) of the forms

$$
\begin{gathered}
\frac{1}{d} \frac{\partial \theta}{\partial y_{1}} \sim-\frac{1}{d} \frac{y_{2}}{|y|^{2}} \\
\left(-\frac{2 \sigma^{2}}{d \gamma^{2}}\right) y_{1} \frac{\partial^{2} \theta}{\partial y_{2}^{2}} \sim \frac{4 \sigma^{2}}{d \gamma^{2}} \frac{y_{1}^{2} y_{2}}{|y|^{4}},
\end{gathered}
$$

which were expressed in local translated coordinates $y$ in (4.12). These two terms will play an important role in determining the dynamical behavior of the vortex helices. Hence, by adding correction terms for cancelation of these two singular terms and also partial fulfilling the boundary conditions in (3.17), we find a good approximate solution defined in (4.43). All of these will be done in Sections 3 and 4. The rest part of the approach is the standard part of the reduction methods. The reader can refer to [13] and [34] and the references therein.

\section{Some PRELIMINARIES}

In this section, we collect some important facts which will be used later. These include the asymptotic behaviors and nondegeneracy of degree one vortex.

Here, we consider the high dimensional case $N \geq 3$. Setting

$$
u=\frac{m_{1}+i m_{2}}{1+m_{3}} \text { and } c=\delta|\log \epsilon|
$$

problem (1.4) becomes

$$
i \delta \epsilon \log \frac{1}{\epsilon} \frac{\partial u}{\partial s_{N}}+\Delta u+\frac{1-|u|^{2}}{1+|u|^{2}} u=\frac{2 \bar{u}}{1+|u|^{2}} \nabla u \cdot \nabla u .
$$

Here and throughout the paper, we use $\bar{u}$ to denote the conjugate of $u$. The parameter $\delta$ is a constant to be chosen in (3.10).

When $\delta=0$ and $N=2$, problem (2.1) admits solutions of standard vortex of degree +1 , i.e. $u=w^{+}:=\rho(\ell) e^{i \theta}$ in polar coordinate $(\ell, \theta)$, where $\rho$ satisfies

$$
\rho^{\prime \prime}+\frac{\rho^{\prime}}{\ell}-\frac{2 \rho\left(\rho^{\prime}\right)^{2}}{1+\rho^{2}}+\left(1-\frac{1}{\ell^{2}}\right) \frac{1-\rho^{2}}{1+\rho^{2}} \rho=0 .
$$

Another solution $w^{-}:=\rho(\ell) e^{-i \theta}$ will be of vortex of degree -1 . These two functions will be our block elements for future construction of approximate solutions.

Natation: For simplicity, from now on, we use $w=\rho(\ell) e^{i \theta}$ to denote the degree +1 vortex. We also assume that $\varrho \in(0,1)$ is a fixed and small constant.

The following properties of $\rho$ are proved in [25].

Lemma 2.1. There hold the asymptotic behaviors:

(1) $\rho(0)=0, \quad 0<\rho(\ell)<1, \quad \rho^{\prime}>0$ for $\ell>0$,

(2) $\rho(\ell)=1-c_{0} \ell^{-1 / 2} e^{-\ell}+O\left(\ell^{-3 / 2} e^{-\ell}\right)$ as $\ell \rightarrow+\infty$, where $c_{0}>0$. 
Setting $w=w_{1}+i w_{2}$ and $z=x_{1}+i x_{2}$, then we need to study the following linearized operator of $\mathbb{S}_{0}$ in $(3.20)$ around the standard profile $w$ :

$$
\begin{aligned}
\mathbb{L}_{0}(\phi)= & \Delta \phi \\
& -\frac{4\left(w_{1} \nabla w_{1}+w_{2} \nabla w_{2}\right)}{1+|w|^{2}} \nabla \phi-\frac{4 \nabla\langle w, \phi\rangle}{1+|w|^{2}} \nabla w \\
& -\frac{4\left(1+|\nabla\rangle w_{1} \nabla w_{1}+w_{2} \nabla w_{2}\right)}{\left(1+|w|^{2}\right)^{2}} \nabla w+\frac{4\langle\nabla w, \nabla \phi\rangle}{1+|w|^{2}} w \\
& -\frac{2|\nabla w|^{2}}{1+|w|^{2}} \phi+\frac{1-|w|^{2}}{1+|w|^{2}} \phi
\end{aligned}
$$

The nondegeneracy of $w$ is contained in the following lemma [34].

Lemma 2.2. Suppose that

$$
\mathbb{L}_{0}[\phi]=0,
$$

where $\phi=i w \psi$, and $\psi=\psi_{1}+i \psi_{2}$ satisfies the following decaying estimates

$$
\begin{aligned}
& \left|\psi_{1}\right|+|z|\left|\nabla \psi_{1}\right| \leq C(1+|z|)^{-\varrho} \\
& \left|\psi_{2}\right|+|z|\left|\nabla \psi_{2}\right| \leq C(1+|z|)^{-1-\varrho}
\end{aligned}
$$

for some $0<\varrho<1$. Then

$$
\phi=c_{1} \frac{\partial w}{\partial x_{1}}+c_{2} \frac{\partial w}{\partial x_{2}}
$$

for certain real constants $c_{1}, c_{2}$.

\section{Symmetric Formulation OF THE PROBlem}

For simplicity of notation, we here only consider the case $N=3$ in the sequel. Recall that by setting

$$
u=\frac{m_{1}+i m_{2}}{1+m_{3}} \quad \text { and } \quad c=\delta|\log \epsilon|,
$$

we have written problem (1.4) in the form (cf. (2.1))

$$
i \delta \epsilon \log \frac{1}{\epsilon} \frac{\partial u}{\partial s_{3}}+\Delta u+\frac{1-|u|^{2}}{1+|u|^{2}} u=\frac{2 \bar{u}}{1+|u|^{2}} \nabla u \cdot \nabla u .
$$

Here and throughout the paper, we use $\bar{u}$ to denote the conjugate of $u$. The parameter $\delta$ is a constant to be chosen in (3.10).

By using the cylinder coordinates $s_{1}=r \cos \Psi, s_{2}=r \sin \Psi, s_{3}=s_{3}$, equation (3.2) is transformed to

$$
i \delta \epsilon \log \frac{1}{\epsilon} \frac{\partial u}{\partial s_{3}}+\frac{\partial^{2} u}{\partial r^{2}}+\frac{1}{r} \frac{\partial u}{\partial r}+\frac{1}{r^{2}} \frac{\partial^{2} u}{\partial \Psi^{2}}+\frac{\partial^{2} u}{\partial s_{3}^{2}}+\frac{1-|u|^{2}}{1+|u|^{2}} u=\frac{2 \bar{u}}{1+|u|^{2}} \nabla u \cdot \nabla u .
$$

For problem (3.3), we want to find a solution $u$ which has a vortex helix directed along the curve in the form

with two parameters

$$
\alpha \in \mathbb{R} \mapsto(d \cos \alpha, d \sin \alpha, \lambda \alpha) \in \mathbb{R}^{3},
$$

$$
d=\frac{\hat{d}}{\epsilon}>0, \quad \lambda=\frac{\hat{\lambda}}{\epsilon} \neq 0 .
$$

Moreover, $u$ is also invariant under the screw motion

$$
\left(r, \Psi, s_{3}\right) \mapsto\left(r, \Psi+\alpha, s_{3}+\lambda \alpha\right), \quad \forall \alpha \in \mathbb{R} .
$$

Hence, $u$ has the symmetry

$$
u\left(r, \Psi, s_{3}\right)=u\left(r, \Psi+\alpha, s_{3}+\lambda \alpha\right)=u\left(r, 0, s_{3}-\lambda \Psi\right),
$$


and also satisfies the problem

$$
\begin{aligned}
& i \delta \epsilon \log \frac{1}{\epsilon} \frac{\partial u}{\partial s_{3}}+\frac{\partial^{2} u}{\partial r^{2}}+\frac{1}{r} \frac{\partial u}{\partial r}+\left(1+\frac{\lambda^{2}}{r^{2}}\right) \frac{\partial^{2} u}{\partial s_{3}^{2}}+\frac{1-|u|^{2}}{1+|u|^{2}} u \\
& =\frac{2 \bar{u}}{1+|u|^{2}}\left[\left(\frac{\partial u}{\partial r}\right)^{2}+\left(1+\frac{\lambda^{2}}{r^{2}}\right)\left(\frac{\partial u}{\partial s_{3}}\right)^{2}\right],
\end{aligned}
$$

which will be defined on the region $\left\{\left(r, s_{3}\right) \in[0, \infty) \times(-\lambda \pi, \lambda \pi)\right\}$.

Before going further, we provide a remark to show the method for the higher dimensional case.

Remark 3.1. We assume that $N=2 m+3$ with integer $m \geq 1$. By steorographic projection, we can write problem (1.4) in the form (cf. (2.1))

$$
i \delta \epsilon \log \frac{1}{\epsilon} \frac{\partial u}{\partial s_{N}}+\Delta u+\frac{1-|u|^{2}}{1+|u|^{2}} u=\frac{2 \bar{u}}{1+|u|^{2}} \nabla u \cdot \nabla u \quad \text { in } \mathbb{R}^{N} .
$$

According to [12], there are helix submanifolds in $\mathbb{R}^{N}$. For the expressions of helix submanifolds, we introduce the coordinates

$$
\begin{aligned}
\left(s_{1}, \cdots, s_{m+1}\right) & =\frac{r}{\sqrt{2}}(\check{\xi} \cos \Psi-\hat{\xi} \sin \Psi), \\
\left(s_{1}, \cdots, s_{2 m+2}\right) & =\frac{r}{\sqrt{2}}(\check{\xi} \sin \Psi+\hat{\xi} \cos \Psi), \\
s_{2 m+3} & =s_{2 m+3},
\end{aligned}
$$

where $\left(r, \Psi, \check{\xi}, \hat{\xi}, s_{2 m+3}\right) \in \mathbb{R} \times \mathbb{R} \times \mathbb{S}^{m} \times \mathbb{S}^{m} \times \mathbb{R}$. Note that $\check{\xi}, \hat{\xi} \in \mathbb{S}^{m}$ are vectors in $\mathbb{R}^{m+1}$. The metric in $\mathbb{R}^{N}$ can be expressed in the form

$$
\mathrm{d} r^{2}+r^{2} \mathrm{~d} \Psi^{2}+\frac{r^{2}}{2} \mathrm{~d} \check{\xi}^{2}+\frac{r^{2}}{2} \mathrm{~d} \hat{\xi}^{2} .
$$

Whence, we get the expressions of Beltrami-Laplace operator

$$
\triangle=\frac{\partial^{2}}{\partial r^{2}}+\frac{2 m+1}{r} \frac{\partial}{\partial r}+\frac{1}{r^{2}} \frac{\partial^{2}}{\partial \Psi^{2}}+\frac{2}{r^{2}} \triangle_{\check{\xi}}+\frac{2}{r^{2}} \triangle_{\hat{\xi}}
$$

where $\triangle_{\xi}$ and $\triangle_{\hat{\xi}}$ are Beltrami-Laplace operators on $\mathbb{S}^{m}$.

Equation (3.8) is transformed to

$$
\begin{aligned}
i \delta \epsilon \log \frac{1}{\epsilon} \frac{\partial u}{\partial s_{N}}+\frac{\partial^{2} u}{\partial r^{2}}+\frac{2 m+1}{r} \frac{\partial u}{\partial r}+\frac{1}{r^{2}} & \frac{\partial^{2} u}{\partial \Psi^{2}}+\frac{2}{r^{2}} \triangle_{\xi}+\frac{2}{r^{2}} \triangle_{\hat{\xi}}+\frac{\partial^{2} u}{\partial s_{N}^{2}} \\
+\frac{1-|u|^{2}}{1+|u|^{2}} u & =\frac{2 \bar{u}}{1+|u|^{2}} \nabla u \cdot \nabla u .
\end{aligned}
$$

For problem (3.9), we want to find a solution $u$, which has a vortex on the helix submanifold in the form

$$
\alpha \in \mathbb{R} \mapsto(d(\check{\xi} \cos \alpha-\hat{\xi} \sin \alpha), d(\check{\xi} \sin \alpha+\hat{\xi} \cos \alpha), \lambda \alpha) \in \mathbb{R}^{N},
$$

where $\check{\xi}, \hat{\xi} \in \mathbb{S}^{m}$ are vectors in $\mathbb{R}^{m+1}$ and the two parameters are

$$
d=\frac{\hat{d}}{\epsilon}>0, \quad \lambda=\frac{\hat{\lambda}}{\epsilon} \neq 0 .
$$

Moreover, $u$ is also invariant under the screw motion

$$
\left(r, \Psi, \check{\xi}, \hat{\xi}, s_{N}\right) \mapsto\left(r, \Psi+\alpha, \check{\xi}, \hat{\xi}, s_{N}+\lambda \alpha\right), \quad \forall \alpha \in \mathbb{R} .
$$

Hence, $u$ has the symmetry

$$
u\left(r, \Psi, \check{\xi}, \hat{\xi}, s_{N}\right)=u\left(r, \Psi+\alpha, \check{\xi}, \hat{\xi}, s_{N}+\lambda \alpha\right)=u\left(r, 0, \check{\xi}_{0}, \hat{\xi}_{0}, s_{N}-\lambda \Psi\right),
$$

where $\check{\xi}_{0}, \hat{\xi}_{0}$ are the north pole of $\mathbb{S}^{m}$. This will derive an equation like (3.7). 
We go back to the case of dimension $N=3$. Recall the parameters in (3.4) and then set

$$
\sigma=\frac{\hat{\lambda}}{\hat{d}}, \quad \gamma=\sqrt{1+\sigma^{2}}, \quad \delta=\frac{1}{\sqrt{\hat{d}^{2}+\hat{\lambda}^{2}}}=\frac{1}{\hat{d} \gamma} .
$$

It is worth mentioning that these three positive parameters are of independence of $\epsilon$. For further convenience of notation, we also introduce the rescaling

$$
\left(r, s_{3}\right)=\left(x_{1}, \gamma x_{2}\right), \quad z=x_{1}+i x_{2} .
$$

Thus (3.7) becomes

$$
\begin{gathered}
i \delta \epsilon \log \frac{1}{\epsilon} \gamma^{-1} \frac{\partial u}{\partial x_{2}}+\frac{\partial^{2} u}{\partial x_{1}^{2}}+\frac{1}{x_{1}} \frac{\partial u}{\partial x_{1}}+\left(1+\frac{\lambda^{2}}{x_{1}^{2}}\right) \gamma^{-2} \frac{\partial^{2} u}{\partial x_{2}^{2}}+\frac{1-|u|^{2}}{1+|u|^{2}} u \\
=\frac{2 \bar{u}}{1+|u|^{2}}\left[\left(\frac{\partial u}{\partial x_{1}}\right)^{2}+\left(1+\frac{\lambda^{2}}{x_{1}^{2}}\right) \gamma^{-2}\left(\frac{\partial u}{\partial x_{2}}\right)^{2}\right] .
\end{gathered}
$$

By using the symmetries, in the sequel, we shall consider the problem on the region

$$
\mathfrak{S}=\left\{\left(x_{1}, x_{2}\right): x_{1} \in \mathbb{R}, x_{2} \in(-\lambda \pi / \gamma, \lambda \pi / \gamma)\right\},
$$

and then impose the boundary conditions

$$
\begin{gathered}
|u(z)| \rightarrow 1 \quad \text { as }\left|x_{1}\right| \rightarrow+\infty, \\
\frac{\partial u}{\partial x_{1}}\left(0, x_{2}\right)=0, \quad \forall x_{2} \in(-\lambda \pi / \gamma, \lambda \pi / \gamma), \\
u\left(x_{1},-\lambda \pi / \gamma\right)=u\left(x_{1}, \lambda \pi / \gamma\right), \quad \forall x_{1} \in \mathbb{R}, \\
u_{x_{2}}\left(x_{1},-\lambda \pi / \gamma\right)=u_{x_{2}}\left(x_{1}, \lambda \pi / \gamma\right), \quad \forall x_{1} \in \mathbb{R} .
\end{gathered}
$$

Before finishing this section, some words are in order to explain the strategies of solving problem (3.12) with boundary conditions in (3.14). It is easy to see that problem(3.12) is invariant under the following two transformations

$$
u(z) \rightarrow \overline{u(\bar{z})}, \quad u(z) \rightarrow u(-\bar{z}) .
$$

Thus we impose the following symmetry on the solution $u$

$$
\Pi:=\{u(z)=\overline{u(\bar{z})}, u(z)=u(-\bar{z})\} .
$$

This symmetry will play an important role in our analysis. As a conclusion, if we write

$$
u\left(x_{1}, x_{2}\right)=u_{1}\left(x_{1}, x_{2}\right)+i u_{2}\left(x_{1}, x_{2}\right),
$$

then $u_{1}$ and $u_{2}$ enjoy the following conditions:

$$
\begin{aligned}
& u_{1}\left(x_{1}, x_{2}\right)=u_{1}\left(-x_{1}, x_{2}\right), \quad u_{1}\left(x_{1}, x_{2}\right)=u_{1}\left(x_{1},-x_{2}\right), \\
& u_{2}\left(x_{1}, x_{2}\right)=u_{2}\left(-x_{1}, x_{2}\right), \quad u_{2}\left(x_{1}, x_{2}\right)=-u_{2}\left(x_{1},-x_{2}\right) \text {, } \\
& \frac{\partial u_{1}}{\partial x_{1}}\left(0, x_{2}\right)=0, \quad \frac{\partial u_{2}}{\partial x_{1}}\left(0, x_{2}\right)=0, \\
& u_{1}\left(x_{1},-\lambda \pi / \gamma\right)=u_{1}\left(x_{1}, \lambda \pi / \gamma\right), \quad \frac{\partial u_{2}}{\partial x_{2}}\left(x_{1},-\lambda \pi / \gamma\right)=\frac{\partial u_{2}}{\partial x_{2}}\left(x_{1}, \lambda \pi / \gamma\right), \\
& \frac{\partial u_{1}}{\partial x_{2}}\left(x_{1},-\lambda \pi / \gamma\right)=\frac{\partial u_{1}}{\partial x_{2}}\left(x_{1}, \lambda \pi / \gamma\right)=0, \quad u_{2}\left(x_{1},-\lambda \pi / \gamma\right)=u_{2}\left(x_{1}, \lambda \pi / \gamma\right)=0 .
\end{aligned}
$$

Problem (3.12)-(3.14) becomes a two-dimensional problem with symmetries defined in (3.16). The key point is then to construct a solution with a vortex of degree +1 at $(d, 0)$ and its antipair of degree -1 at $(-d, 0)$. Additional to the computations for standard vortices in two dimensional case, there are two extra derivative terms

$$
\frac{1}{x_{1}} \frac{\partial u}{\partial x_{1}} \quad \text { and } \quad \gamma^{-2}\left(1+\frac{\lambda^{2}}{x_{1}^{2}}\right) \frac{\partial^{2} u}{\partial x_{2}^{2}} .
$$


Moreover, we shall improve the approximate solution to satisfy the boundary conditions in (3.17). To the end of constructing vortex pairs locating at $(d, 0)$ and $(-d, 0)$, we write

$$
\begin{gathered}
\frac{\partial^{2} u}{\partial x_{1}^{2}}+\left(1+\frac{\lambda^{2}}{x_{1}^{2}}\right) \gamma^{-2} \frac{\partial^{2} u}{\partial x_{2}^{2}}=\Delta+\gamma^{-2}\left[\frac{\lambda^{2}}{x_{1}^{2}}-\frac{\lambda^{2}}{d^{2}}\right] \frac{\partial^{2} u}{\partial x_{2}^{2}}, \\
\left(\frac{\partial u}{\partial x_{1}}\right)^{2}+\left(1+\frac{\lambda^{2}}{x_{1}^{2}}\right) \gamma^{-2}\left(\frac{\partial u}{\partial x_{2}}\right)^{2}=\nabla u \cdot \nabla u+\gamma^{-2}\left[\frac{\lambda^{2}}{x_{1}^{2}}-\frac{\lambda^{2}}{d^{2}}\right]\left(\frac{\partial u}{\partial x_{2}}\right)^{2},
\end{gathered}
$$

and then set

$$
\begin{aligned}
& \mathbb{S}_{0}[u]:=\Delta u+F(u)-\frac{2 \bar{u}}{1+|u|^{2}} \nabla u \cdot \nabla u, \\
& \mathbb{S}_{1}[u]:=-\frac{2 \bar{u}}{1+|u|^{2}} \gamma^{-2}\left[\frac{\lambda^{2}}{x_{1}^{2}}-\frac{\lambda^{2}}{d^{2}}\right]\left(\frac{\partial u}{\partial x_{2}}\right)^{2}, \\
& \mathbb{S}_{2}[u]:=\gamma^{-2}\left[\frac{\lambda^{2}}{x_{1}^{2}}-\frac{\lambda^{2}}{d^{2}}\right] \frac{\partial^{2} u}{\partial x_{2}^{2}}, \\
& \mathbb{S}_{3}[u]:=\frac{1}{x_{1}} \frac{\partial u}{\partial x_{1}}, \quad \mathbb{S}_{4}[u]:=i \delta \gamma^{-1} \epsilon|\log \epsilon| \frac{\partial u}{\partial x_{2}}, \\
& \mathbb{S}[u]:=\mathbb{S}_{0}[u]+\mathbb{S}_{1}[u]+\mathbb{S}_{2}[u]+\mathbb{S}_{3}[u]+\mathbb{S}_{4}[u] .
\end{aligned}
$$

In the above $F(u)=\frac{1-|u|^{2}}{1+|u|^{2}} u$.

It is worth mentioning that problem (3.12) is also degenerate when $x_{1}=0$ due to the term

$$
\gamma^{-2}\left(1+\frac{\lambda^{2}}{x_{1}^{2}}\right) \frac{\partial^{2} u}{\partial x_{2}^{2}}
$$

We need more analysis to handle the singularity as $x_{1} \rightarrow 0$. Whence, for the convenience of further careful analysis, we divide the region $\mathfrak{S}$ (cf. (3.13)) into three parts:

$$
\begin{aligned}
& \mathfrak{S}_{+}=\left\{\left(x_{1}, x_{2}\right): x_{1}>2 \varpi / \epsilon,-\lambda \pi / \gamma<x_{2}<\lambda \pi / \gamma\right\}, \\
& \mathfrak{S}_{0}=\left\{\left(x_{1}, x_{2}\right):-2 \varpi / \epsilon<x_{1}<2 \varpi / \epsilon,-\lambda \pi / \gamma<x_{2}<\lambda \pi / \gamma\right\}, \\
& \mathfrak{S}_{-}=\left\{\left(x_{1}, x_{2}\right): x_{1}<-2 \varpi / \epsilon,-\lambda \pi / \gamma<x_{2}<\lambda \pi / \gamma\right\} .
\end{aligned}
$$

Here $\varpi$ is a small positive constant less than $1 / 1000$.

\section{Approximate solutions}

4.1. First Approximate Solution and its Error. Recall the vortex solutions $w^{+}$and $w^{-}$ defined in (2.2), and also the parameters in (3.4). Define the smooth cut-off function $\tilde{\eta}$ in the form

$$
\tilde{\eta}(s)=1 \quad \text { for }|s| \leq 1, \quad \tilde{\eta}(s)=0 \quad \text { for } \quad|s| \geq 2 .
$$

For the convenience of notation, for $\vec{e}_{1}=(1,0)$ and any given $\left(x_{1}, x_{2}\right) \in \mathbb{R}^{2}$, let $\theta_{d \vec{e}_{1}}$ and $\theta_{-d \vec{e}_{1}}$ be respectively the angle arguments of the vectors $x-d \vec{e}_{1}=\left(x_{1}-d, x_{2}\right)$ and $x+d \vec{e}_{1}=\left(x_{1}+d, x_{2}\right)$ in the $\left(x_{1}, x_{2}\right)$ plane. We also let

$$
\ell_{1}\left(x_{1}, x_{2}\right)=\sqrt{\left(x_{1}-d\right)^{2}+x_{2}^{2}}, \quad \ell_{2}\left(x_{1}, x_{2}\right)=\sqrt{\left(x_{1}+d\right)^{2}+x_{2}^{2}},
$$

be the distance functions between the point $\left(x_{1}, x_{2}\right)$ and the pair of vortices locating at the points $d \vec{e}_{1}$ and $-d \vec{e}_{1}$. We also write

$$
\tilde{\rho}=\rho\left(\ell_{1}\right) \rho\left(\ell_{2}\right), \quad \varphi_{0}=\theta_{d \vec{e}_{1}}-\theta_{-d \vec{e}_{1}} .
$$


For each fixed $d:=\hat{d} / \epsilon$ with $\hat{d} \in[1 / 100,100]$, we define the first approximate solution

$$
v_{0}(z):=\eta_{\epsilon}\left(x_{1}\right) e^{i \varphi_{0}}+\left(1-\eta_{\epsilon}\left(x_{1}\right)\right) \tilde{\rho} e^{i \varphi_{0}},
$$

where $\eta_{\epsilon}\left(x_{1}\right)=\tilde{\eta}\left(\epsilon\left|x_{1}\right| / \varpi\right)$. As in (3.21), here $\varpi$ is the small positive constant less than $1 / 1000$. A simple computation shows that

$$
\left|v_{0}(z)\right| \rightarrow 1 \text { as }|z| \rightarrow+\infty
$$

In fact, for $|z|>>d$, we have

$$
\begin{aligned}
v_{0} & \approx e^{i \theta_{d \vec{e}_{1}}-i \theta_{-d \vec{e}_{1}}} \\
& \approx \frac{\left(x_{1}^{2}-d^{2}+x_{2}^{2}+2 i x_{2} d\right)}{\sqrt{\left(x_{1}-d\right)^{2}+x_{2}^{2}} \sqrt{\left(x_{1}+d\right)^{2}+x_{2}^{2}}} \\
& \approx 1+O\left(\frac{d^{2}}{|z|^{2}}+\frac{d}{|z|}\right) .
\end{aligned}
$$

If $\theta_{\tilde{\xi}}$ denotes the angle argument around $\tilde{\xi}$ and $r_{\tilde{\xi}}=|z-\tilde{\xi}|$, it is easy to see that

$$
\nabla r_{\tilde{\xi}}=\frac{1}{|z-\tilde{\xi}|}(z-\tilde{\xi}), \quad \nabla \theta_{\tilde{\xi}}=\frac{1}{|z-\tilde{\xi}|^{2}}(z-\tilde{\xi})^{\perp}, \quad\left|\nabla \theta_{\tilde{\xi}}\right|=\frac{1}{|z-\tilde{\xi}|},
$$

where we denote $z^{\perp}=\left(-x_{2}, x_{1}\right)$.

Recall the operators defined in (3.20) and the decompositions of $\mathfrak{S}$ in (3.21). One of the main objectives of this subsection is to compute the error $\mathbb{S}\left[v_{0}\right]$. We start the computations on the region $\mathfrak{S}_{+}$. Note that on $\mathfrak{S}_{+}$, there hold $\eta_{\epsilon}=0$ and

$$
v_{0}=\tilde{\rho} e^{i \varphi_{0}} .
$$

Moreover, by our construction and the properties of $\rho$, we have

$$
\begin{aligned}
v_{0} & =w\left(z-d \vec{e}_{1}\right) e^{-i \theta_{-d \vec{e}_{1}}}\left[1+O\left(e^{-d / 2-\ell_{1} / 2}\right)\right], \\
\left|v_{0}\right| & =\rho\left(\left|z-d \vec{e}_{1}\right|\right)\left[1+O\left(e^{-d / 2-\ell_{1} / 2}\right)\right] .
\end{aligned}
$$

We now obtain the following estimates

$$
\begin{gathered}
\Delta v_{0}=\left(\Delta w-2 i \nabla w \cdot \nabla \theta_{-d \vec{e}_{1}}-w \nabla \theta_{-d \vec{e}_{1}} \cdot \nabla \theta_{-d \vec{e}_{1}}+O\left(e^{-d / 2-\ell_{1} / 2}\right)\right) e^{-i \theta_{-d \vec{e}_{1}}} \\
-\frac{2 \bar{v}}{1+|v|^{2}} \nabla v_{0} \cdot \nabla v_{0}=\frac{-2 \bar{w}}{1+\rho^{2}} e^{-i \theta_{-d \vec{e}_{1}}}\left[\nabla w \cdot \nabla w-2 i w \nabla w \cdot \nabla \theta_{-d \vec{e}_{1}}-w^{2} \nabla \theta_{-d \vec{e}_{1}} \cdot \nabla \theta_{-d \vec{e}_{1}}\right] \\
+O\left(e^{-d / 2-\ell_{1} / 2}\right),
\end{gathered}
$$

and then also

$$
\frac{1-\left|v_{0}\right|^{2}}{1+\left|v_{0}\right|^{2}} v_{0}=\frac{1-\rho^{2}}{1+\rho^{2}} w\left(1+O\left(e^{-d / 2-\ell_{1} / 2}\right)\right) e^{-i \theta_{-d \vec{e}_{1}}} .
$$

Combining the estimates above, we have for $z \in \mathfrak{S}_{+}$,

$$
\begin{aligned}
\mathbb{S}_{0}\left[v_{0}\right] & =\left[\frac{2\left(\rho^{2}-1\right)}{\rho^{2}+1} i \nabla w \cdot \nabla \theta_{-d \vec{e}_{1}}+\frac{\rho^{2}-1}{\rho^{2}+1} w \nabla \theta_{-d \vec{e}_{1}} \cdot \nabla \theta_{-d \vec{e}_{1}}+O\left(e^{-d / 2-\ell_{1} / 2}\right)\right] e^{-i \theta_{-d \vec{e}_{1}}} \\
& =\Omega_{10} e^{i \varphi_{0}}
\end{aligned}
$$

where

$$
\begin{aligned}
\Omega_{10} \equiv- & \frac{2 \rho\left(\rho^{2}-1\right)}{\rho^{2}+1} \nabla \theta_{d \vec{e}_{1}} \cdot \nabla \theta_{-d \vec{e}_{1}}+\frac{\rho\left(\rho^{2}-1\right)}{\rho^{2}+1} \nabla \theta_{-d \vec{e}_{1}} \cdot \nabla \theta_{-d \vec{e}_{1}} \\
& +i \frac{2\left(\rho^{2}-1\right)}{\rho^{2}+1} \nabla \rho \cdot \nabla \theta_{-d \vec{e}_{1}}+O\left(e^{-d / 2-\ell_{1} / 2}\right) .
\end{aligned}
$$


The calculation for the terms $\mathbb{S}_{1}\left[v_{0}\right]$ and $\mathbb{S}_{2}\left[v_{0}\right]$ are proceeded as

$$
\begin{aligned}
\mathbb{S}_{1}\left[v_{0}\right] & =-\frac{2 \bar{v}_{0}}{1+\left|v_{0}\right|^{2}} \gamma^{-2}\left[\frac{\lambda^{2}}{x_{1}^{2}}-\frac{\lambda^{2}}{d^{2}}\right]\left\{\frac{\partial}{\partial x_{2}}\left(\tilde{\rho} e^{i \varphi_{0}}\right)\right\}^{2} \\
& =-\frac{2 \tilde{\rho}}{1+|\tilde{\rho}|^{2}} \gamma^{-2}\left[\frac{\lambda^{2}}{x_{1}^{2}}-\frac{\lambda^{2}}{d^{2}}\right]\left(\left|\frac{\partial \tilde{\rho}}{\partial x_{2}}\right|^{2}-|\tilde{\rho}|^{2}\left|\frac{\partial \varphi_{0}}{\partial x_{2}}\right|^{2}+2 i \tilde{\rho} \frac{\partial \tilde{\rho}}{\partial x_{2}} \frac{\partial \varphi_{0}}{\partial x_{2}}\right) e^{i \varphi_{0}}
\end{aligned}
$$

and

$$
\begin{aligned}
\mathbb{S}_{2}\left[v_{0}\right]= & \gamma^{-2}\left[\frac{\lambda^{2}}{x_{1}^{2}}-\frac{\lambda^{2}}{d^{2}}\right] \frac{\partial^{2}}{\partial x_{2}^{2}}\left[\tilde{\rho} e^{i \varphi_{0}}\right] \\
= & \gamma^{-2}\left[\frac{\lambda^{2}}{x_{1}^{2}}-\frac{\lambda^{2}}{d^{2}}\right]\left[\frac{\partial^{2} \tilde{\rho}}{\partial x_{2}^{2}}+2 i \frac{\partial \tilde{\rho}}{\partial x_{2}} \frac{\partial \varphi_{0}}{\partial x_{2}}-\tilde{\rho}\left|\frac{\partial \varphi_{0}}{\partial x_{2}}\right|^{2}\right] e^{i \varphi_{0}} \\
& +i v_{0} \gamma^{-2}\left[\frac{\lambda^{2}}{x_{1}^{2}}-\frac{\lambda^{2}}{d^{2}}\right] \frac{\partial^{2} \varphi_{0}}{\partial x_{2}^{2}} .
\end{aligned}
$$

Whence, there holds

$$
\mathbb{S}_{1}\left[v_{0}\right]+\mathbb{S}_{2}\left[v_{0}\right]=\Omega_{11} e^{i \varphi_{0}}+i v_{0} \mathbb{S}_{2}\left[\varphi_{0}\right]
$$

where

$$
\begin{aligned}
\Omega_{11} \equiv & \gamma^{-2}\left[\frac{\lambda^{2}}{x_{1}^{2}}-\frac{\lambda^{2}}{d^{2}}\right]\left(-\frac{2 \tilde{\rho}}{1+|\tilde{\rho}|^{2}}\left|\frac{\partial \tilde{\rho}}{\partial x_{2}}\right|^{2}+\frac{\partial^{2} \tilde{\rho}}{\partial x_{2}^{2}}\right) \\
& +\gamma^{-2}\left[\frac{\lambda^{2}}{x_{1}^{2}}-\frac{\lambda^{2}}{d^{2}}\right] \frac{1-|\tilde{\rho}|^{2}}{1+|\tilde{\rho}|^{2}}\left[-\tilde{\rho}\left|\frac{\partial \varphi_{0}}{\partial x_{2}}\right|^{2}+2 i \frac{\partial \tilde{\rho}}{\partial x_{2}} \frac{\partial \varphi_{0}}{\partial x_{2}}\right] .
\end{aligned}
$$

We here need more analysis on the last term $i v_{0} \mathbb{S}_{2}\left[\varphi_{0}\right]$ in the above formula. Note that

$$
\frac{\partial^{2} \varphi_{0}}{\partial x_{2}^{2}}=\frac{\partial^{2} \theta_{d \vec{e}_{1}}}{\partial x_{2}^{2}}-\frac{\partial^{2} \theta_{-d \vec{e}_{1}}}{\partial x_{2}^{2}}=\frac{-2\left(x_{1}-d\right) x_{2}}{\ell_{1}^{4}}-\frac{-2\left(x_{1}+d\right) x_{2}}{\ell_{2}^{4}} .
$$

In the neighborhood of $d \vec{e}_{1}$ there holds

$$
\begin{aligned}
\gamma^{-2}\left[\frac{\lambda^{2}}{x_{1}^{2}}-\frac{\lambda^{2}}{d^{2}}\right] & =-\frac{2 \sigma^{2}}{d \gamma^{2}}\left(x_{1}-d\right)+\frac{\sigma^{2}}{d \gamma^{2}} \frac{3 d\left(x_{1}-d\right)^{2}+2\left(x_{1}-d\right)^{3}}{x_{1}^{2}} \\
& =-\frac{2 \sigma^{2}}{d \gamma^{2}}\left(x_{1}-d\right)+O\left(\left(x_{1}-d\right)^{2} / d^{2}\right) .
\end{aligned}
$$

Whence there is a singularity in $\mathbb{S}_{2}\left[\varphi_{0}\right]$ of the form

$$
-\frac{2 \sigma^{2}}{d \gamma^{2}}\left(x_{1}-d\right) \frac{\partial^{2} \theta_{d \vec{e}_{1}}}{\partial x_{2}^{2}}=-\frac{2 \sigma^{2}}{d \gamma^{2}} y_{1} \frac{\partial^{2} \theta_{d \vec{e}_{1}}}{\partial y_{2}^{2}}=\frac{4 \sigma^{2}}{d \gamma^{2}} \frac{y_{1}^{2} y_{2}}{|y|^{4}}
$$

with variable

$$
y=x-d \vec{e}_{1} .
$$

A similar singularity exists in the neighborhood of $-d \vec{e}_{1}$

$$
\frac{2 \sigma^{2}}{d \gamma^{2}}\left(x_{1}+d\right) \frac{\partial^{2} \theta_{-d \vec{e}_{1}}}{\partial x_{2}^{2}}=-\frac{2 \sigma^{2}}{d \gamma^{2}} \tilde{y}_{1} \frac{\partial^{2} \theta_{-d \vec{e}_{1}}}{\partial \tilde{y}_{2}^{2}}=-\frac{4 \sigma^{2}}{d \gamma^{2}} \frac{\tilde{y}_{1}^{2} \tilde{y}_{2}}{|\tilde{y}|^{4}}
$$

with the variable $\tilde{y}=x+d \vec{e}_{1}$. 
The term $\mathbb{S}_{3}\left[v_{0}\right]$ obeys the following asymptotic behavior

$$
\begin{aligned}
\mathbb{S}_{3}\left[v_{0}\right] & =\frac{x_{1}-d}{x_{1} \ell_{1}} \frac{\rho^{\prime}\left(\ell_{1}\right)}{\rho\left(\ell_{1}\right)} v_{0}+\frac{x_{1}+d}{x_{1} \ell_{2}} \frac{\rho^{\prime}\left(\ell_{2}\right)}{\rho\left(\ell_{2}\right)} v_{0}+i v_{0} \frac{1}{x_{1}} \frac{\partial \varphi_{0}}{\partial x_{1}} \\
& \equiv \Omega_{12} e^{i \varphi_{0}}+i v_{0} \mathbb{S}_{3}\left[\varphi_{0}\right]
\end{aligned}
$$

where

$$
\Omega_{12}=\frac{x_{1}-d}{x_{1} \ell_{1}} \rho^{\prime}\left(\ell_{1}\right) \rho\left(\ell_{2}\right)+\frac{x_{1}+d}{x_{1} \ell_{2}} \rho^{\prime}\left(\ell_{2}\right) \rho\left(\ell_{1}\right) .
$$

By the computation

$$
\mathbb{S}_{3}\left[\varphi_{0}\right]=\frac{1}{x_{1}} \frac{\partial \varphi_{0}}{\partial x_{1}}=\frac{1}{x_{1}} \frac{\partial \theta_{d \vec{e}_{1}}}{\partial x_{1}}-\frac{1}{x_{1}} \frac{\partial \theta_{-d \vec{e}_{1}}}{\partial x_{1}}=\frac{1}{x_{1}}\left(\frac{-x_{2}}{\ell_{1}^{2}}+\frac{x_{2}}{\ell_{2}^{2}}\right),
$$

we find that it is a singular term. More precisely, in the neighborhood of $d \vec{e}_{1}$ with variable $y=x-d \vec{e}_{1}$, there is a singularity in $\mathbb{S}_{3}\left[\varphi_{0}\right]$ of the form

$$
\frac{1}{x_{1}} \frac{\partial \theta_{d \vec{e}_{1}}}{\partial x_{1}}=\left[\frac{1}{d}-\frac{y_{1}}{d\left(d+y_{1}\right)}\right] \frac{\partial \theta_{d \vec{e}_{1}}}{\partial y_{1}} \quad \text { with } \quad \frac{1}{d} \frac{\partial \theta_{d \vec{e}_{1}}}{\partial y_{1}}=-\frac{1}{d} \frac{y_{2}}{|y|^{2}} .
$$

A similar singularity exists in the neighborhood of $-d \vec{e}_{1}$ with the variable $\tilde{y}=x+d \vec{e}_{1}$

$$
-\frac{1}{x_{1}} \frac{\partial \theta_{-d \vec{e}_{1}}}{\partial x_{1}}=\left[-\frac{1}{d}+\frac{\tilde{y}_{1}}{d\left(\tilde{y}_{1}-d\right)}\right](-1) \frac{\partial \theta_{-d \vec{e}_{1}}}{\partial \tilde{y}_{1}} \quad \text { with } \quad \frac{1}{d} \frac{\partial \theta_{-d \vec{e}_{1}}}{\partial \tilde{y}_{1}}=-\frac{1}{d} \frac{\tilde{y}_{2}}{|\tilde{y}|^{2}} \text {. }
$$

We can estimate the term in $\mathbb{S}_{4}\left[v_{0}\right]$ as follows

$$
i \delta \gamma^{-1} \epsilon \log \frac{1}{\epsilon} \frac{\partial v_{0}}{\partial x_{2}}=i \delta \gamma^{-1} \epsilon \log \frac{1}{\epsilon} \frac{\partial}{\partial x_{2}}\left[\rho\left(\ell_{1}\right) e^{i \varphi_{0}}+O\left(e^{-d / 2-\ell_{1} / 2}\right)\right]=\Omega_{13} e^{i \varphi_{0}}
$$

where

$$
\Omega_{13} \equiv i \delta \gamma^{-1} \epsilon \log \frac{1}{\epsilon}\left[\frac{\partial \rho\left(\ell_{1}\right)}{\partial x_{2}}+i \rho\left(\ell_{1}\right) \frac{\partial \varphi_{0}}{\partial x_{2}}\right]+O\left(\epsilon \log \frac{1}{\epsilon} e^{-d / 2-\ell_{1} / 2}\right)
$$

In summary, we have obtained for $z \in \mathfrak{S}_{+}$

$$
\begin{aligned}
\mathbb{S}\left[v_{0}\right] & =\mathbb{S}_{0}\left[v_{0}\right]+\mathbb{S}_{1}\left[v_{0}\right]+\mathbb{S}_{2}\left[v_{0}\right]+\mathbb{S}_{3}\left[v_{0}\right]+\mathbb{S}_{4}\left[v_{0}\right] \\
& =\sum_{i=1}^{3} \Omega_{1 i} e^{i \varphi_{0}}+i v_{0} \mathbb{S}_{2}\left[\varphi_{0}\right]+i v_{0} \mathbb{S}_{3}\left[\varphi_{0}\right]
\end{aligned}
$$

A similar (and almost identical) estimate also holds in the region $\mathfrak{S}_{-}$. For the convenience of later use, we denote that for $z \in \mathfrak{S}_{+} \cup \mathfrak{S}_{-}$

$$
\hat{E} \equiv \mathbb{S}\left[v_{0}\right]-i v_{0} \mathbb{S}_{2}\left[\varphi_{0}\right]-i v_{0} \mathbb{S}_{3}\left[\varphi_{0}\right]=\sum_{i=0}^{3} \Omega_{1 i} e^{i \varphi_{0}}
$$

We now compute the error on $\mathfrak{S}_{0}$. In this region, we only do the computation on the region $\left\{-\varpi / \epsilon<x_{1}<\varpi / \epsilon\right\}$, where $\eta_{\epsilon}=1$ and $v_{0}=e^{i \varphi_{0}}$. Note that $\mathbb{S}_{0}\left[v_{0}\right]=0$. On the other hand

$$
\begin{aligned}
\mathbb{S}_{1}\left[v_{0}\right]+\mathbb{S}_{2}\left[v_{0}\right] & \equiv \gamma^{-2}\left[\frac{\lambda^{2}}{x_{1}^{2}}-\frac{\lambda^{2}}{d^{2}}\right]\left(-\frac{2 \bar{v}_{0}}{1+\left|v_{0}\right|^{2}}\left(\frac{\partial v_{0}}{\partial x_{2}}\right)^{2}+\frac{\partial^{2} v_{0}}{\partial x_{2}^{2}}\right) \\
& =i v_{0} \gamma^{-2}\left[\frac{\lambda^{2}}{x_{1}^{2}}-\frac{\lambda^{2}}{d^{2}}\right] \frac{\partial^{2} \varphi_{0}}{\partial x_{2}^{2}} .
\end{aligned}
$$


We give more explicit computations

$$
\begin{aligned}
\frac{\partial^{2} \varphi_{0}}{\partial x_{2}^{2}}= & \frac{-2\left(x_{1}-d\right) x_{2}}{\ell_{1}^{4}}-\frac{-2\left(x_{1}+d\right) x_{2}}{\ell_{2}^{4}} \\
= & \frac{4 d x_{2}}{\left(d^{2}+x_{2}^{2}\right)^{2}}-\frac{8 x_{2} x_{1}^{2} d\left(\ell_{1}+\ell_{2}\right)}{\ell_{1}^{4} \ell_{2}^{4}} \\
& -\frac{2 x_{2} x_{1}^{2} d}{\ell_{1}^{4} \ell_{2}^{4}\left(d^{2}+x_{2}^{2}\right)^{2}}\left[\ell_{1}^{4}\left(d^{2}+x_{2}^{2}+\ell_{2}^{2}\right)+\ell_{2}^{4}\left(d^{2}+x_{2}^{2}+\ell_{1}^{2}\right)\right] \\
& +\frac{16 x_{2} x_{1}^{2} d^{3}}{\ell_{1}^{4} \ell_{2}^{4}\left(d^{2}+x_{2}^{2}\right)}\left[\left(\ell_{1}^{2}+\ell_{2}^{2}\right)\left(d^{2}+x_{2}^{2}\right)+\ell_{2}^{2} \ell_{1}^{2}\right] .
\end{aligned}
$$

We now write

$$
\mathbb{S}_{1}\left[v_{0}\right]+\mathbb{S}_{2}\left[v_{0}\right] \equiv \Omega_{4}+i v_{0} \mathbb{S}_{2}\left[\arctan \left(x_{2} / d\right)\right] .
$$

Here if $x_{1}$ approaches 0 , there is another singular term in the form

$$
i v_{0} \mathbb{S}_{2}\left[\arctan \left(x_{2} / d\right)\right]=i v_{0} \gamma^{-2}\left[\frac{\lambda^{2}}{x_{1}^{2}}-\frac{\lambda^{2}}{d^{2}}\right] \frac{4 d x_{2}}{\left(d^{2}+x_{2}^{2}\right)^{2}} .
$$

In the next subsection, we will introduce a correction in the phase term to get rid of these three mentioned singularities.

The term $\mathbb{S}_{3}\left[v_{0}\right]$ on $\mathfrak{S}_{0}$ obeys the following asymptotic behavior

$$
\mathbb{S}_{3}\left[v_{0}\right]=\frac{1}{x_{1}} \frac{\partial v_{0}}{\partial x_{1}}=i v_{0} \frac{1}{x_{1}} \frac{\partial \varphi_{0}}{\partial x_{1}} .
$$

By the computation

$$
\frac{1}{x_{1}} \frac{\partial \varphi_{0}}{\partial x_{1}}=\frac{1}{x_{1}} \frac{\partial \theta_{d \vec{e}_{1}}}{\partial x_{1}}-\frac{1}{x_{1}} \frac{\partial \theta_{-d \vec{e}_{1}}}{\partial x_{1}}=\frac{1}{x_{1}}\left(\frac{-x_{2}}{\ell_{1}^{2}}+\frac{x_{2}}{\ell_{2}^{2}}\right)=\frac{4 d x_{2}}{\ell_{1}^{2} \ell_{2}^{2}} .
$$

Hence, due to the fact that $v_{0}$ is an even term on the variable $x_{1}$, one can check that $\mathbb{S}_{3}\left[v_{0}\right]$ does not contain singular components as $x_{1} \rightarrow 0$.

We can estimate the term in $\mathbb{S}_{4}\left[v_{0}\right]$ on $\mathfrak{S}_{0}$ as follows

$$
\begin{aligned}
i \delta \gamma^{-1} \epsilon \log \frac{1}{\epsilon} \frac{\partial v_{0}}{\partial x_{2}} & =-\delta \gamma^{-1} \epsilon \log \frac{1}{\epsilon} v_{0} \frac{\partial \varphi_{0}}{\partial x_{2}} \\
& =\delta \gamma^{-1} \epsilon \log \frac{1}{\epsilon} v_{0}\left[\frac{\partial \theta_{d \vec{e}_{1}}}{\partial x_{2}}-\frac{\partial \theta_{-d \vec{e}_{1}}}{\partial x_{2}}\right] \\
& =O\left(\epsilon^{2}|\log \epsilon|\right),
\end{aligned}
$$

due the facts

$$
\begin{gathered}
\frac{\partial \theta_{d \vec{e}_{1}}}{\partial x_{2}}=\frac{x_{1}-d}{\left(x_{1}-d\right)^{2}+x_{2}^{2}}=O\left(d^{-1}\right)=O(\epsilon), \\
\frac{\partial \theta_{-d \vec{e}_{1}}}{\partial x_{2}}=\frac{x_{1}+d}{\left(x_{1}+d\right)^{2}+x_{2}^{2}}=O\left(d^{-1}\right)=O(\epsilon) .
\end{gathered}
$$

Adding all terms gives that, for $z \in \mathfrak{S}_{0}$

$$
\mathbb{S}\left[v_{0}\right]=\Omega_{4}+i v_{0} \mathbb{S}_{2}\left[\arctan \left(x_{2} / d\right)\right]+i v_{0} \frac{4 d x_{2}}{\ell_{1}^{2} \ell_{2}^{2}}-\delta \gamma^{-1} \epsilon \log \frac{1}{\epsilon} v_{0} \frac{\partial \varphi_{0}}{\partial x_{2}} .
$$

Here we also denote for $z \in \mathfrak{S}_{0}$

$$
\hat{E}=\mathbb{S}\left[v_{0}\right]-i v_{0} \mathbb{S}_{2}\left[\arctan \left(x_{2} / d\right)\right]
$$


As a conclusion, a direct application of the above computations yields the decay estimates for the error $\hat{E}$.

Lemma 4.1. It holds that for $z \in\left(B_{2}\left(d \vec{e}_{1}\right) \cup B_{2}\left(-d \vec{e}_{1}\right)\right)^{c} \cap \mathfrak{S}$

$$
\begin{gathered}
\left|\operatorname{Re}\left(\hat{E} /\left(i v_{0}\right)\right)\right| \leq \frac{C \epsilon^{1-\varrho}}{\left(1+\ell_{1}\right)^{3}}+\frac{C \epsilon^{1-\varrho}}{\left(1+\ell_{2}\right)^{3}}, \\
\left|\operatorname{Im}\left(\hat{E} /\left(i v_{0}\right)\right)\right| \leq \frac{C \epsilon^{1-\varrho}}{\left(1+\ell_{1}\right)^{1+\varrho}}+\frac{C \epsilon^{1-\varrho}}{\left(1+\ell_{2}\right)^{1+\varrho}},
\end{gathered}
$$

where $\varrho \in(0,1)$ is a constant.

Proof: Let us derive the estimate for $\Omega_{13} e^{i \varphi_{0}} /\left(i v_{0}\right)$ on $\mathfrak{S}_{+}$

$$
\begin{aligned}
\Omega_{13} e^{i \varphi_{0}} /\left(i v_{0}\right)= & \delta \gamma^{-1} \epsilon \log \frac{1}{\epsilon} \frac{\partial \rho\left(\ell_{1}\right)}{\partial x_{2}} \frac{1}{\rho\left(\ell_{1}\right)}+i \delta \gamma^{-1} \epsilon \log \frac{1}{\epsilon} \frac{\partial \varphi_{0}}{\partial x_{2}} \\
& +\epsilon \log \frac{1}{\epsilon} O\left(e^{-\ell_{1}}+e^{-\ell_{2}}\right) \\
= & \epsilon \log \frac{1}{\epsilon} O\left(e^{-\ell_{1}}+e^{-\ell_{2}}\right)+i \delta \gamma^{-1} \epsilon \log \frac{1}{\epsilon}\left[\frac{x_{1}-d}{\ell_{1}^{2}}-\frac{x_{1}+d}{\ell_{2}^{2}}\right] .
\end{aligned}
$$

The estimate for $\Omega_{13} e^{i \varphi_{0}} /\left(i v_{0}\right)$ like (4.27) then follows. Let us also notice that for $z \in \mathfrak{S}_{+}$with $\ell_{1}<d$

$$
\epsilon \log \frac{1}{\epsilon}\left|\frac{x_{1}-d}{\ell_{1}^{2}}-\frac{x_{1}+d}{\ell_{2}^{2}}\right| \leq \frac{C \epsilon \log \frac{1}{\epsilon}}{\left(1+\ell_{1}\right)} \leq \frac{C \epsilon^{1-\varrho}}{\left(1+\ell_{1}\right)^{1+\varrho}} .
$$

On the other hand, for $z \in \mathfrak{S}_{+}$with $\ell_{1}>d$, we then have

$$
\epsilon \log \frac{1}{\epsilon}\left|\frac{x_{1}-d}{\ell_{1}^{2}}-\frac{x_{1}+d}{\ell_{2}^{2}}\right| \leq \frac{C \epsilon \log \frac{1}{\epsilon}}{\left(1+\ell_{1}\right)^{2}} \leq \frac{C \epsilon^{1-\varrho}}{\left(1+\ell_{1}\right)^{1+\varrho}} .
$$

Thus the estimate for $\Omega_{13} e^{i \varphi_{0}} /\left(i v_{0}\right)$ like (4.28) is proved. Similarly, the estimates for other terms in $\hat{E}$ follow from (4.7), (4.8), (4.14).

4.2. Further Improvement of the Approximation. As we promised in previous subsection, we now define a new correction $\varphi_{d}$ in the phase term formally by

$$
\varphi_{d}(z)=\varphi_{s}(z)+\varphi_{1}(z)+\varphi_{2}(z)
$$

Then we will define an improved approximation in (4.43) and estimate its error by substituting it into (3.12) in Section 4.3.

Now we give the definitions of all terms in $\varphi_{d}$. By recalling the cut-off function $\eta_{\epsilon}\left(x_{1}\right)$ in (4.4) we set

$$
\varphi_{2}=2 \eta_{\epsilon}\left(x_{1}\right) \arctan \left(x_{2} / d\right)
$$

to cancel the singular term in (4.23), in such a way that

$$
\begin{aligned}
\Delta \varphi_{2} & =2 \eta_{\epsilon}\left(x_{1}\right) \frac{\partial^{2}}{\partial x_{2}^{2}} \arctan \left(x_{2} / d\right)+O\left(\epsilon^{2}\right) \\
& =-\eta_{\epsilon}\left(x_{1}\right) \frac{4 d x_{2}}{\left(d^{2}+x_{2}^{2}\right)^{2}}+O\left(\epsilon^{2}\right) .
\end{aligned}
$$

To cancel the singularities in (4.16) and (4.11) rewritten in the form

$$
\frac{y_{2}}{d|y|^{2}}-\frac{4 \sigma^{2}}{d \gamma^{2}} \frac{y_{1}^{2} y_{2}}{|y|^{4}}=\frac{y_{2}}{d \gamma^{2}|y|^{2}}-\frac{\sigma^{2}\left(3 y_{1}^{2}-y_{2}^{2}\right) y_{2}}{d \gamma^{2}|y|^{4}},
$$


we want to find a function $\Phi\left(y_{1}, y_{2}\right)$ by solving the problem in the translated coordinates $\left(y_{1}, y_{2}\right)$

$$
\frac{\partial^{2} \Phi}{\partial y_{1}^{2}}+\frac{\partial^{2} \Phi}{\partial y_{2}^{2}}=\frac{y_{2}}{d \gamma^{2}|y|^{2}}-\frac{\sigma^{2}\left(3 y_{1}^{2}-y_{2}^{2}\right) y_{2}}{d \gamma^{2}|y|^{4}} \text { in } \mathbb{R}^{2}
$$

In fact, we can solve this problem by separation of variables and then obtain

$$
\Phi\left(y_{1}, y_{2}\right)=\frac{1}{4 d \gamma^{2}} y_{2} \log |y|^{2}-\frac{\sigma^{2}}{2 d \gamma^{2}} \frac{y_{2}^{3}}{|y|^{2}}+\frac{3 \sigma^{2}}{8 d \gamma^{2}} y_{2} .
$$

Let $\chi$ be a smooth cut-off function in a way such that $\chi(\vartheta)=1$ for $\vartheta<\hat{d} / 10$ and $\chi(\vartheta)=0$ for $\vartheta>\hat{d} / 5$. The singular part in $\varphi_{d}$ is defined by

$$
\begin{aligned}
\varphi_{s}(z):= & \left(\chi\left(\epsilon \ell_{1}\right)+\chi\left(\epsilon \ell_{2}\right)\right)\left[\frac{x_{2}}{4 d \gamma^{2}} \log \ell_{1}^{2}-\frac{x_{2}}{4 d \gamma^{2}} \log \ell_{2}^{2}\right] \\
& -\left(\chi\left(\epsilon \ell_{1}\right)+\chi\left(\epsilon \ell_{2}\right)\right)\left[\frac{\sigma^{2} x_{2}^{3}}{2 d \gamma^{2} \ell_{1}^{2}}-\frac{\sigma^{2} x_{2}^{3}}{2 d \gamma^{2} \ell_{2}^{2}}\right] .
\end{aligned}
$$

For later use, we compute:

$$
\begin{aligned}
\frac{\partial \varphi_{s}}{\partial x_{1}}= & \epsilon\left(\chi^{\prime}\left(\epsilon \ell_{1}\right) \frac{x_{1}-d}{\ell_{1}}+\chi^{\prime}\left(\epsilon \ell_{2}\right) \frac{x_{1}+d}{\ell_{2}}\right)\left[\frac{x_{2}}{4 d \gamma^{2}} \log \left(\ell_{1}\right)^{2}-\frac{x_{2}}{4 d \gamma^{2}} \log \left(\ell_{2}\right)^{2}\right] \\
& +\left(\chi\left(\epsilon \ell_{1}\right)+\chi\left(\epsilon \ell_{2}\right)\right)\left[\frac{x_{2}\left(x_{1}-d\right)}{2 d \gamma^{2} \ell_{1}^{2}}-\frac{x_{2}\left(x_{1}+d\right)}{2 d \gamma^{2} \ell_{2}^{2}}\right] \\
& -\epsilon\left(\chi^{\prime}\left(\epsilon \ell_{1}\right) \frac{x_{1}-d}{\ell_{1}}+\chi^{\prime}\left(\epsilon \ell_{2}\right) \frac{x_{1}+d}{\ell_{2}}\right)\left[\frac{\sigma^{2} x_{2}^{3}}{2 d \gamma^{2} \ell_{1}^{2}}-\frac{\sigma^{2} x_{2}^{3}}{2 d \gamma^{2} \ell_{2}^{2}}\right] \\
& +\left(\chi\left(\epsilon \ell_{1}\right)+\chi\left(\epsilon \ell_{2}\right)\right)\left[\frac{\sigma^{2} x_{2}^{3}\left(x_{1}-d\right)}{d \gamma^{2} \ell_{1}^{4}}-\frac{\sigma^{2} x_{2}^{3}\left(x_{1}+d\right)}{d \gamma^{2} \ell_{2}^{4}}\right], \\
\frac{\partial \varphi_{s}}{\partial x_{2}}=\epsilon\left(\chi^{\prime}\left(\epsilon \ell_{1}\right) \frac{x_{2}}{\ell_{1}}+\chi^{\prime}\left(\epsilon \ell_{2}\right) \frac{x_{2}}{\ell_{2}}\right)\left[\frac{x_{2}}{4 d \gamma^{2}} \log \left(\ell_{1}\right)^{2}-\frac{x_{2}}{4 d \gamma^{2}} \log \left(\ell_{2}\right)^{2}\right] & \left(\chi\left(\epsilon \ell_{1}\right)+\chi\left(\epsilon \ell_{2}\right)\right)\left[\frac{1}{4 d \gamma^{2}} \log \left(\ell_{1}\right)^{2}-\frac{1}{4 d \gamma^{2}} \log \left(\ell_{2}\right)^{2}+\frac{x_{2}^{2}}{2 d \gamma^{2} \ell_{1}^{2}}-\frac{x_{2}^{2}}{2 d \gamma^{2} \ell_{2}^{2}}\right] \\
+ & \epsilon\left(\chi^{\prime}\left(\epsilon \ell_{1}\right) \frac{x_{2}}{\ell_{1}}+\chi^{\prime}\left(\epsilon \ell_{2}\right) \frac{x_{2}}{\ell_{2}}\right)\left[-\frac{\sigma^{2} x_{2}^{3}}{2 d \gamma^{2} \ell_{1}^{2}}+\frac{\sigma^{2} x_{2}^{3}}{2 d \gamma^{2} \ell_{2}^{2}}\right] \\
+ & \left(\chi\left(\epsilon \ell_{1}\right)+\chi\left(\epsilon \ell_{2}\right)\right)\left[-\frac{3 \sigma^{2} x_{2}^{2}}{2 d \gamma^{2} \ell_{1}^{2}}+\frac{3 \sigma^{2} x_{2}^{2}}{2 d \gamma^{2} \ell_{2}^{2}}+\frac{\sigma^{2} x_{2}^{4}}{d \gamma^{2} \ell_{1}^{4}}-\frac{\sigma^{2} x_{2}^{4}}{d \gamma^{2} \ell_{2}^{4}}\right] .
\end{aligned}
$$

Hence, we obtain

$$
\begin{aligned}
\nabla \varphi_{s}= & \chi\left(\epsilon \ell_{1}\right)\left[\frac{1}{2 d \gamma^{2}}\left(0, \log \ell_{1}\right)+O\left(\epsilon \log \ell_{1}\right)\right] \\
& -\chi\left(\epsilon \ell_{2}\right)\left[\frac{1}{2 d \gamma^{2}}\left(0, \log \ell_{2}\right)+O\left(\epsilon \log \ell_{2}\right)\right] .
\end{aligned}
$$

Note that the function $\varphi_{s}$ is continuous but $\nabla \varphi_{s}$ is not. The singularity of $\varphi_{s}$ comes from its derivatives.

While by recalling the operators $\mathbb{S}_{2}, \mathbb{S}_{3}$ in $(3.20)$, the region $\mathfrak{S}$ in (3.13) and its decompositions in (3.21), we find the term $\varphi_{1}(z)$ by solving the problem

$$
\begin{aligned}
{\left[\Delta+\mathbb{S}_{2}+\mathbb{S}_{3}\right] \varphi_{1} } & =-\left[\Delta+\mathbb{S}_{2}+\mathbb{S}_{3}\right]\left(\varphi_{0}+\varphi_{s}+\varphi_{2}\right) \text { in } \mathfrak{S}, \\
\varphi_{1} & =-\varphi_{0}-\varphi_{s}-\varphi_{2} \text { on } \partial \mathfrak{S} .
\end{aligned}
$$


We derive the estimate of $\varphi_{1}$ by computing the right hand side of (4.38). Note that

$$
\left[\Delta+\mathbb{S}_{2}+\mathbb{S}_{3}\right]\left(\varphi_{0}+\varphi_{s}+\varphi_{2}\right)=\Delta\left(\varphi_{s}+\varphi_{2}\right)+\left[\mathbb{S}_{2}+\mathbb{S}_{3}\right]\left(\varphi_{0}+\varphi_{s}+\varphi_{2}\right),
$$

due to $\Delta \varphi_{0}=0$. Recall the formulas (4.9), (4.10), (4.11), (4.15), (4.16). For $z \in B_{\frac{d}{10}}\left(d \vec{e}_{1}\right)$, there hold

$$
\begin{aligned}
& \Delta \varphi_{s}+\left[\mathbb{S}_{2}+\mathbb{S}_{3}\right] \varphi_{0} \\
& =-\frac{x_{1}-d}{d x_{1}} \frac{\partial \theta_{d \vec{e}_{1}}}{\partial x_{1}}+\frac{\sigma^{2}}{\gamma^{2}} \frac{3 d\left(x_{1}-d\right)^{2}+2\left(x_{1}-d\right)^{3}}{d x_{1}^{2}} \frac{\partial^{2} \theta_{d \vec{e}_{1}}}{\partial x_{2}^{2}}+O\left(d^{-2}\right) \\
& =-\frac{x_{1}-d}{d x_{1}} \frac{x_{2}}{\ell_{1}^{2}}-\frac{\sigma^{2}}{\gamma^{2}} \frac{3 d\left(x_{1}-d\right)^{2}+2\left(x_{1}-d\right)^{3}}{d x_{1}^{2}} \frac{\left(x_{1}-d\right) x_{2}}{\ell_{1}^{4}}+O\left(d^{-2}\right) \\
& =O\left(d^{-2}\right)=O\left(\epsilon^{2}\right) .
\end{aligned}
$$

We also get for $z \in B_{\frac{d}{10}}\left(d \vec{e}_{1}\right)$

$$
\left[\Delta+\mathbb{S}_{2}+\mathbb{S}_{3}\right] \varphi_{2}=0
$$

due to $\varphi_{2}=0$ in this region. Similarly, by recalling (4.10) and (4.36), we obtain for $z \in B_{\frac{d}{10}}\left(d \vec{e}_{1}\right)$

$$
\begin{aligned}
\mathbb{S}_{2}\left[\varphi_{s}\right]= & {\left[-\frac{2 \sigma^{2}}{d \gamma^{2}}\left(x_{1}-d\right)+O\left(\left(x_{1}-d\right)^{2} / d^{2}\right)\right] } \\
& \times\left(\chi\left(\epsilon \ell_{1}\right)+\chi\left(\epsilon \ell_{2}\right)\right)\left\{\frac{3\left(1-2 \sigma^{2}\right) x_{2}}{2 d \gamma^{2} \ell_{1}^{2}}-\frac{\left(1+\sigma^{2}\right) x_{2}^{3}}{d \gamma^{2} \ell_{1}^{4}}-\frac{2 \sigma^{2} x_{2}^{5}}{d \gamma^{2} \ell_{2}^{6}}\right\}+O\left(\epsilon^{2}\right) \\
= & O\left(\epsilon^{2}\right),
\end{aligned}
$$

and by recalling (4.35)

$$
\begin{aligned}
\mathbb{S}_{3}\left[\varphi_{s}\right]= & \frac{1}{x_{1}} \frac{1}{2 d \gamma^{2}}\left[\frac{x_{2}\left(x_{1}-d\right)}{\ell_{1}^{2}}-\frac{x_{2}\left(x_{1}+d\right)}{\ell_{2}^{2}}\right] \\
& +\frac{1}{x_{1}} \frac{\sigma^{2}}{2 d \gamma^{2}}\left[\frac{x_{2}^{3}\left(x_{1}-d\right)}{\ell_{1}^{4}}-\frac{x_{2}^{3}\left(x_{1}+d\right)}{\ell_{2}^{4}}\right]+O\left(\epsilon^{2}\right) \\
= & O\left(\epsilon^{2}\right) .
\end{aligned}
$$

For $z \in B_{\frac{d}{10}}\left(-d \vec{e}_{1}\right)$, it is easy to see that we also get $O\left(\epsilon^{2}\right)$. For $z$ in the region

$$
\left(\mathfrak{S}_{+} \cup \mathfrak{S}_{-}\right)-\left(B_{\frac{d}{5}}\left(d \vec{e}_{1}\right) \cup B_{\frac{d}{5}}\left(-d \vec{e}_{1}\right)\right),
$$

we have $\varphi_{s}=\varphi_{2}=0$ and then

$$
\begin{aligned}
{\left[\Delta+\mathbb{S}_{2}+\mathbb{S}_{3}\right]\left(\varphi_{0}+\varphi_{s}+\varphi_{2}\right) } & =\gamma^{-2}\left[\frac{\lambda^{2}}{x_{1}^{2}}-\frac{\lambda^{2}}{d^{2}}\right] \frac{\partial^{2} \varphi_{0}}{\partial x_{2}^{2}}+\frac{1}{x_{1}} \frac{\partial \varphi_{0}}{\partial x_{1}} \\
& =-\gamma^{-2}\left[\frac{\lambda^{2}}{x_{1}^{2}}-\frac{\lambda^{2}}{d^{2}}\right]\left(\frac{2 x_{2}\left(x_{1}-d\right)}{\ell_{1}^{4}}-\frac{2 x_{2}\left(x_{1}+d\right)}{\ell_{2}^{4}}\right)+\frac{-4 x_{2}}{\ell_{1}^{2} \ell_{2}^{2}}
\end{aligned}
$$

By recalling (4.21), (4.23) and (4.24) and (4.31), we do the computation on the region $\mathfrak{S}_{0}$. There also hold $\varphi_{s}=0$ and so

$$
\begin{aligned}
& {\left[\Delta+\mathbb{S}_{2}+\mathbb{S}_{3}\right]\left(\varphi_{0}+\varphi_{s}+\varphi_{2}\right)} \\
& =\Delta \varphi_{2}+\mathbb{S}_{3}\left[\varphi_{0}\right]+\mathbb{S}_{2}\left[\varphi_{0}+\varphi_{2}\right] \\
& =\frac{4 d x_{2}}{\left(d^{2}+x_{2}^{2}\right)^{2}}+\frac{4 d x_{2}}{\ell_{1}^{2} \ell_{2}^{2}}+O\left(\epsilon^{2}\right)
\end{aligned}
$$




$$
\begin{aligned}
+\gamma^{-2}\left[\frac{\lambda^{2}}{x_{1}^{2}}-\frac{\lambda^{2}}{d^{2}}\right]\{ & -\frac{8 x_{2} x_{1}^{2} d\left(\ell_{1}+\ell_{2}\right)}{\ell_{1}^{4} \ell_{2}^{4}}-\frac{16 x_{2} x_{1}^{2} d^{3}}{\ell_{1}^{4} \ell_{2}^{4}\left(d^{2}+x_{2}^{2}\right)}\left(\left(\ell_{1}^{2}+\ell_{2}^{2}\right)\left(d^{2}+x_{2}^{2}\right)+\ell_{2}^{2} \ell_{1}^{2}\right) \\
& \left.-\frac{2 x_{2} x_{1}^{2} d}{\ell_{1}^{4} \ell_{2}^{4}\left(d^{2}+x_{2}^{2}\right)^{2}}\left(\ell_{1}^{4}\left(d^{2}+x_{2}^{2}+\ell_{2}^{2}\right)+\ell_{2}^{4}\left(d^{2}+x_{2}^{2}+\ell_{1}^{2}\right)\right)\right\} .
\end{aligned}
$$

Whence, going back to the original variable $\left(r, s_{3}\right)$ in $(3.3)$ and letting $\hat{\varphi}\left(r, s_{3}\right)=\varphi_{1}(z)$ we see that

$$
\left|\Delta_{r, s_{3}} \hat{\varphi}+\mathbb{S}_{2}[\hat{\varphi}]+\mathbb{S}_{3}[\hat{\varphi}]\right| \leq \frac{C}{\left(\sqrt{1+r^{2}+\left|s_{3}\right|^{2}}\right)^{3}} .
$$

Thus we can choose $\varphi_{1}$ such that

$$
\hat{\varphi}=O\left(\frac{1}{\sqrt{1+r^{2}+\left|s_{3}\right|^{2}}}\right) .
$$

The regular term $\varphi_{1}$ is $C^{1}$ in the original variable $\left(r, s_{3}\right)$.

We observe also that by our definitions, the function

$$
\tilde{\varphi}:=\varphi_{0}+\varphi_{d}
$$

satisfies

$$
\left[\Delta+\mathbb{S}_{2}+\mathbb{S}_{3}\right] \tilde{\varphi}=0 \quad \text { on } \quad \mathfrak{S}, \quad \tilde{\varphi}=0 \quad \text { on } \quad \partial \mathfrak{S} .
$$

From the decomposition of $\varphi_{d}$, we will see that the singular term contains $x_{2} \log \ell_{1}$ which becomes dominant when we calculate the speed of traveling wave in Section 7.

Finally, we define an improved approximation

$$
V_{d}(z):=\eta_{\epsilon}\left(x_{1}\right) e^{i\left(\varphi_{0}+\varphi_{d}\right)}+\left(1-\eta_{\epsilon}\left(x_{1}\right)\right) \rho\left(\ell_{1}\right) \rho\left(\ell_{2}\right) e^{i\left(\varphi_{0}+\varphi_{d}\right)} .
$$

4.3. Error Estimates. Recall the notation in (4.3) and (4.41). We write $V_{d}$ in (4.43) in the form

$$
V_{d}(z)=\eta_{\epsilon}\left(x_{1}\right) e^{i \tilde{\varphi}}+\left(1-\eta_{\epsilon}\left(x_{1}\right)\right) \tilde{\rho} e^{i \tilde{\varphi}}, \quad \forall z=x_{1}+i x_{2} \in \mathfrak{S} .
$$

We shall check that $V_{d}$ is a good approximate solution in the sense that it satisfies the conditions in (3.17) and has a small error.

It is easy to show that

$$
V_{d}(z)=\overline{V_{d}(\bar{z})}, \quad V_{d}(z)=V_{d}(-\bar{z}), \quad \frac{\partial V_{d}}{\partial x_{1}}\left(0, x_{2}\right)=0 .
$$

Recall the boundary condition in (4.42). It is obvious that

$$
\operatorname{Im} V_{d}=\left[\eta_{\epsilon}\left(x_{1}\right)+\left(1-\eta_{\epsilon}\left(x_{1}\right)\right) \tilde{\rho}\right] \sin \tilde{\varphi}=0 \quad \text { on } \partial \mathfrak{S},
$$

and

$$
\begin{aligned}
\frac{\partial \operatorname{Re} V_{d}}{\partial x_{2}} & =\frac{\partial}{\partial x_{2}}\left[\eta_{\epsilon}\left(x_{1}\right) \cos \tilde{\varphi}+\left(1-\eta_{\epsilon}\left(x_{1}\right)\right) \tilde{\rho} \cos \tilde{\varphi}\right] \\
& =\frac{\partial \tilde{\rho}}{\partial x_{2}}\left(1-\eta_{\epsilon}\left(x_{1}\right)\right) \cos \tilde{\varphi} \text { on } \partial \mathfrak{S} .
\end{aligned}
$$

It can be checked that $V_{d}$ satisfies the conditions in (3.17) except

$$
\frac{\partial V_{d}}{\partial x_{2}}=0 \quad \text { on } \quad \partial \mathfrak{S}
$$

Let us start to compute the error by plugging $V_{d}$ into (3.12). We start the computations on the region $\mathfrak{S}_{+}$and $\mathfrak{S}_{-}$. Note that there holds

$$
V_{d}=v_{0} e^{i \varphi_{d}} .
$$


It implies that

$$
\begin{aligned}
\Delta V_{d} & =\left(\Delta v_{0}-\left|\nabla \varphi_{d}\right|^{2} v_{0}+2 i \nabla v_{0} \cdot \nabla \varphi_{d}+i v_{0} \Delta \varphi_{d}\right) e^{i \varphi_{d}} \\
& =\left[\Delta v_{0}-\left|\nabla \varphi_{d}\right|^{2} v_{0}+2 i \nabla v_{0} \cdot \nabla \varphi_{d}-i v_{0}\left(\mathbb{S}_{2}[\tilde{\varphi}]+\mathbb{S}_{3}[\tilde{\varphi}]\right)\right] e^{i \varphi_{d}}
\end{aligned}
$$

Here we have used the relation $\Delta \varphi_{0}=0$ and the equation in (4.42). We continue to compute other terms:

$$
\begin{aligned}
-\frac{2 \bar{V}_{d}}{1+\left|V_{d}\right|^{2}} \nabla V_{d} \cdot \nabla V_{d} & =-\frac{2 \bar{v}_{0}}{1+\left|v_{0}\right|^{2}}\left[\nabla v_{0} \cdot \nabla v_{0}-v_{0}^{2}\left|\nabla \varphi_{d}\right|^{2}+2 i v_{0} \nabla v_{0} \cdot \nabla \varphi_{d}\right] e^{i \varphi_{d}} \\
& =-\frac{2 \bar{v}_{0}}{1+\left|v_{0}\right|^{2}} \nabla v_{0} \cdot \nabla v_{0} e^{i \varphi_{d}}+\frac{2 \tilde{\rho}^{2}}{1+|\tilde{\rho}|^{2}}\left[v_{0}\left|\nabla \varphi_{d}\right|^{2}-2 i \nabla v_{0} \cdot \nabla \varphi_{d}\right] e^{i \varphi_{d}}
\end{aligned}
$$

We then obtain that

$$
\mathbb{S}_{0}\left[V_{d}\right]=\mathbb{S}_{0}\left[v_{0}\right] e^{i \varphi_{d}}-i v_{0}\left(\mathbb{S}_{2}[\tilde{\varphi}]+\mathbb{S}_{3}[\tilde{\varphi}]\right) e^{i \varphi_{d}}+\Omega_{20} e^{i \tilde{\varphi}}
$$

where we have defined

$$
\Omega_{20}=\left[\frac{\tilde{\rho}\left(\tilde{\rho}^{2}-1\right)}{\tilde{\rho}^{2}+1}\left|\nabla \varphi_{d}\right|^{2}-2 \frac{\left(1-\tilde{\rho}^{2}\right) \tilde{\rho}}{1+\tilde{\rho}^{2}} \nabla \varphi_{0} \cdot \nabla \varphi_{d}+2 i \frac{1-\tilde{\rho}^{2}}{1+\tilde{\rho}^{2}} \nabla \tilde{\rho} \cdot \nabla \varphi_{d}\right] .
$$

In a small neighborhood of $d \vec{e}_{1}$, we write

$$
\begin{aligned}
\mathbb{S}_{1}\left[V_{d}\right]+\mathbb{S}_{2}\left[V_{d}\right]= & -\frac{2 \bar{V}_{d}}{1+\left|V_{d}\right|^{2}} \gamma^{-2}\left[\frac{\lambda^{2}}{x_{1}^{2}}-\frac{\lambda^{2}}{d^{2}}\right]\left(\frac{\partial V_{d}}{\partial x_{2}}\right)^{2}+\gamma^{-2}\left[\frac{\lambda^{2}}{x_{1}^{2}}-\frac{\lambda^{2}}{d^{2}}\right] \frac{\partial^{2} V_{d}}{\partial x_{2}^{2}} \\
= & -\gamma^{-2}\left(\frac{\lambda^{2}}{x_{1}^{2}}-\frac{\lambda^{2}}{d^{2}}\right) \frac{2 \bar{v}_{0}}{1+\left|v_{0}\right|^{2}}\left[\left(\frac{\partial v_{0}}{\partial x_{2}}\right)^{2}+2 i v_{0} \frac{\partial v_{0}}{\partial x_{2}} \frac{\partial \varphi_{d}}{\partial x_{2}}-v_{0}^{2}\left(\frac{\partial \varphi_{d}}{\partial x_{2}}\right)^{2}\right] e^{i \varphi_{d}} \\
& +\gamma^{-2}\left(\frac{\lambda^{2}}{x_{1}^{2}}-\frac{\lambda^{2}}{d^{2}}\right)\left[\frac{\partial^{2} v_{0}}{\partial x_{2}^{2}}+2 i \frac{\partial v_{0}}{\partial x_{2}} \frac{\partial \varphi_{d}}{\partial x_{2}}+i v_{0} \frac{\partial^{2} \varphi_{d}}{\partial x_{2}^{2}}-v_{0}\left(\frac{\partial \varphi_{d}}{\partial x_{2}}\right)^{2}\right] e^{i \varphi_{d}} \\
= & \left(\mathbb{S}_{1}\left[v_{0}\right]+\mathbb{S}_{2}\left[v_{0}\right]\right) e^{i \varphi_{d}}+i v_{0} \mathbb{S}_{2}\left[\varphi_{d}\right] e^{i \varphi_{d}}+\Omega_{21} e^{i \tilde{\varphi}}
\end{aligned}
$$

where

$$
\begin{aligned}
\Omega_{21}=\gamma^{-2}\left(\frac{\lambda^{2}}{x_{1}^{2}}-\frac{\lambda^{2}}{d^{2}}\right)[ & \frac{\left(|\tilde{\rho}|^{2}-1\right) \tilde{\rho}}{1+|\tilde{\rho}|^{2}}\left(\frac{\partial \varphi_{d}}{\partial x_{2}}\right)^{2}-2 \frac{\left(1-|\tilde{\rho}|^{2}\right) \tilde{\rho}}{1+|\tilde{\rho}|^{2}} \frac{\partial \varphi_{0}}{\partial x_{2}} \frac{\partial \varphi_{d}}{\partial x_{2}} \\
& \left.+2 i \frac{1-|\tilde{\rho}|^{2}}{1+|\tilde{\rho}|^{2}} \frac{\partial \tilde{\rho}}{\partial x_{2}} \frac{\partial \varphi_{d}}{\partial x_{2}}\right] .
\end{aligned}
$$

The estimates for the terms $\mathbb{S}_{3}\left[V_{d}\right]$ and $\mathbb{S}_{4}\left[V_{d}\right]$ are expressed as

$$
\mathbb{S}_{3}\left[V_{d}\right]=\frac{1}{x_{1}} \frac{\partial V_{d}}{\partial x_{1}}=\mathbb{S}_{3}\left[v_{0}\right] e^{i \varphi_{d}}+i v_{0} \mathbb{S}_{3}\left[\varphi_{d}\right] e^{i \varphi_{d}}
$$

and

$$
\mathbb{S}_{4}\left[V_{d}\right]=i \delta \gamma^{-1} \epsilon \log \frac{1}{\epsilon} \frac{\partial V_{d}}{\partial x_{2}}=\mathbb{S}_{4}\left[v_{0}\right] e^{i \varphi_{d}}+\Omega_{23} e^{i \tilde{\varphi}}
$$

In the above we also define

$$
\Omega_{23}=-\delta \gamma^{-1} \epsilon \log \frac{1}{\epsilon} \frac{\partial \varphi_{d}}{\partial x_{2}} \tilde{\rho} .
$$

For the convenience of notation, we will set $\Omega_{22}=0$ in the sequel. 
Recall all the components of $\mathbb{S}\left[v_{0}\right]$ in (4.19) and $\hat{E}$ in (4.20). The total error on $\mathfrak{S}_{+}$is

$$
\begin{aligned}
\mathbb{S}\left[V_{d}\right] & =\mathbb{S}\left[v_{0}\right] e^{i \tilde{\varphi}}+i v_{0}\left(-\mathbb{S}_{2}[\tilde{\varphi}]-\mathbb{S}_{3}[\tilde{\varphi}]+\mathbb{S}_{2}\left[\varphi_{d}\right]+\mathbb{S}_{3}\left[\varphi_{d}\right]\right) e^{i \varphi_{d}}+\sum_{i=0}^{3} \Omega_{2 i} e^{i \tilde{\varphi}} \\
& =\mathbb{S}\left[v_{0}\right] e^{i \tilde{\varphi}}-i v_{0}\left(\mathbb{S}_{2}\left[\varphi_{0}\right]+\mathbb{S}_{3}\left[\varphi_{0}\right]\right) e^{i \varphi_{d}}+\sum_{i=0}^{3} \Omega_{2 i} e^{i \tilde{\varphi}} \\
& =\sum_{i=0}^{3}\left(\Omega_{1 i}+\Omega_{2 i}\right) e^{i \tilde{\varphi}} .
\end{aligned}
$$

Note that $\Omega_{i j}$ 's are defined in (4.7), (4.8), (4.14), (4.18), (4.47), (4.48), (4.49). A similar formula also holds on $\mathfrak{S}_{-}$. Setting $z=d \vec{e}_{1}+y$ in $\mathfrak{S}_{+}$, we then have

$$
\nabla \varphi_{s}=-\frac{1}{2 d \gamma^{2}} \log d \nabla y_{2}+O(\epsilon \log |y|), \quad \nabla \varphi_{r}=O(\epsilon)
$$

Thus

$$
\nabla \tilde{\rho} \cdot \nabla \tilde{\varphi}=O\left(\epsilon|\log \epsilon| \rho^{\prime}\right)+O\left(\epsilon \rho^{\prime} \log |y|\right) .
$$

These asymptotic expression will play an important role in the reduction part.

We now compute the error on $\mathfrak{S}_{0}$. In this region, we only do the computation on the region $\left\{-\varpi / \epsilon<x_{1}<\varpi / \epsilon\right\}$, where $\eta_{\epsilon}=1$ and $V_{d}=e^{i \tilde{\varphi}}$. Note that

$$
\mathbb{S}_{0}\left[V_{d}\right]=i V_{d} \Delta \tilde{\varphi}
$$

and

$$
\begin{aligned}
\mathbb{S}_{1}\left[V_{d}\right]+\mathbb{S}_{2}\left[V_{d}\right] & \equiv \gamma^{-2}\left[\frac{\lambda^{2}}{x_{1}^{2}}-\frac{\lambda^{2}}{d^{2}}\right]\left(-\frac{2 \overline{V_{d}}}{1+\left|V_{d}\right|^{2}}\left(\frac{\partial V_{d}}{\partial x_{2}}\right)^{2}+\frac{\partial^{2} V_{d}}{\partial x_{2}^{2}}\right) \\
& =i V_{d} \gamma^{-2}\left[\frac{\lambda^{2}}{x_{1}^{2}}-\frac{\lambda^{2}}{d^{2}}\right] \frac{\partial^{2} \tilde{\varphi}}{\partial x_{2}^{2}} \\
& =i V_{d} \mathbb{S}_{2}[\tilde{\varphi}] .
\end{aligned}
$$

The term $\mathbb{S}_{3}\left[V_{d}\right]$ obeys the following asymptotic behavior

$$
\mathbb{S}_{3}\left[V_{d}\right] \equiv \frac{1}{x_{1}} \frac{\partial V_{d}}{\partial x_{1}}=i V_{d} \frac{1}{x_{1}} \frac{\partial \tilde{\varphi}}{\partial x_{1}}=i V_{d} \mathbb{S}_{3}[\tilde{\varphi}]
$$

We can estimate the term in $\mathbb{S}_{4}\left[V_{d}\right]$ as follows

$$
\begin{aligned}
i \delta \gamma^{-1} \epsilon|\log \epsilon| \frac{\partial V_{d}}{\partial x_{2}} & =-\delta \gamma^{-1} \epsilon|\log \epsilon| V_{d} \frac{\partial \tilde{\varphi}}{\partial x_{2}} \\
& =\delta \gamma^{-1} \epsilon|\log \epsilon| V_{d}\left[\frac{\partial \varphi_{0}}{\partial x_{2}}+\frac{\partial \varphi_{1}}{\partial x_{2}}+\frac{\partial \varphi_{2}}{\partial x_{2}}\right] \\
& =V_{d} O\left(\epsilon^{2}|\log \epsilon|\right),
\end{aligned}
$$

due the facts

$$
\begin{gathered}
\frac{\partial \theta_{d \vec{e}_{1}}}{\partial x_{2}}=\frac{x_{1}-d}{\left(x_{1}-d\right)^{2}+y_{2}^{2}}=O\left(d^{-1}\right)=O(\epsilon), \\
\frac{\partial \theta_{-d \vec{e}_{1}}}{\partial x_{2}}=\frac{x_{1}+d}{\left(x_{1}+d\right)^{2}+y_{2}^{2}}=O\left(d^{-1}\right)=O(\epsilon) .
\end{gathered}
$$

Finally, by combining the above computations and using the equation in (4.42), we get the error $\mathbb{S}\left[V_{d}\right]$ on $\mathfrak{S}_{0}$. 


\section{Setting up of the Problem}

Now we introduce the set-up of the reduction procedure. We look for solutions of (3.12)-(3.14) in the form

where $\eta$ is a function such that

$$
u(z)=\eta(z)\left(V_{d}+i V_{d} \psi\right)+(1-\eta(z)) V_{d} e^{i \psi},
$$

$$
\eta(z)=\tilde{\eta}\left(\ell_{1}\right)+\tilde{\eta}\left(\ell_{2}\right)
$$

See (4.1) for the definition of the cut-off function $\tilde{\eta}$. This nonlinear decomposition (5.1) was introduced first in [13] for Ginzburg-Landau equation.

The conditions imposed on $u$ in (3.14) and (3.16) can be transmitted to the symmetry on $\psi$

$$
\begin{gathered}
\psi(z)=\psi(-\bar{z}), \quad \psi(z)=-\overline{\psi(\bar{z})}, \\
\frac{\partial \psi}{\partial x_{1}}\left(0, x_{2}\right)=0, \quad \psi\left(x_{1},-\lambda \pi / \gamma\right)=\psi\left(x_{1}, \lambda \pi / \gamma\right), \\
{\left.\left[\frac{\partial V_{d}}{\partial x_{2}}+i V_{d} \psi_{x_{2}}\right]\right|_{\left(x_{1},-\lambda \pi / \gamma\right)}=\left.\left[\frac{\partial V_{d}}{\partial x_{2}}+i V_{d} \psi_{x_{2}}\right]\right|_{\left(x_{1}, \lambda \pi / \gamma\right)}}
\end{gathered}
$$

More precisely, by the computations in (4.44) and (4.45), for $\psi=\psi_{1}+i \psi_{2}$, there hold the conditions

$$
\begin{aligned}
\psi_{1}\left(x_{1}, x_{2}\right)=\psi_{1}\left(-x_{1}, x_{2}\right), & \psi_{1}\left(x_{1}, x_{2}\right)=-\psi_{1}\left(x_{1},-x_{2}\right), \\
\psi_{2}\left(x_{1}, x_{2}\right)=\psi_{2}\left(-x_{1}, x_{2}\right), & \psi_{2}\left(x_{1}, x_{2}\right)=\psi_{2}\left(x_{1},-x_{2}\right), \\
\frac{\partial \psi_{1}}{\partial x_{1}}\left(0, x_{2}\right)=0, & \frac{\partial \psi_{2}}{\partial x_{1}}\left(0, x_{2}\right)=0, \\
\psi_{1}\left(x_{1},-\lambda \pi / \gamma\right)=\psi_{1}\left(x_{1}, \lambda \pi / \gamma\right)=0, & \frac{\partial \psi_{1}}{\partial x_{2}}\left(x_{1},-\lambda \pi / \gamma\right)=\frac{\partial \psi_{1}}{\partial x_{2}}\left(x_{1}, \lambda \pi / \gamma\right), \\
\frac{\partial \psi_{2}}{\partial x_{2}}\left(x_{1},-\lambda \pi / \gamma\right)=\frac{1}{\tilde{\rho}} \frac{\partial \tilde{\rho}}{\partial x_{2}}\left(x_{1},-\lambda \pi / \gamma\right), & \frac{\partial \psi_{2}}{\partial x_{2}}\left(x_{1}, \lambda \pi / \gamma\right)=\frac{1}{\tilde{\rho}} \frac{\partial \tilde{\rho}}{\partial x_{2}}\left(x_{1}, \lambda \pi / \gamma\right), \\
\psi_{2}\left(x_{1},-\lambda \pi / \gamma\right)=\psi_{2}\left(x_{1}, \lambda \pi / \gamma\right) . &
\end{aligned}
$$

The symmetry will be important in solving the linear problems in that it excludes all but one kernel. We may write $\psi=\psi_{1}+i \psi_{2}$ with $\psi_{1}, \psi_{2}$ real-valued and then set

$$
u=V_{d}+\phi, \quad \phi=\eta i V_{d} \psi+(1-\eta) V_{d}\left(e^{i \psi}-1\right) .
$$

In the sequel, we will derive the explicit form for the linearized problem.

In the inner region $\left\{z \in B_{1}\left(d \vec{e}_{1}\right) \cup B_{1}\left(-d \vec{e}_{1}\right)\right\}$, we have

$$
u=V_{d}+\phi \text {, }
$$

and the equation for $\phi$ becomes

$$
\mathbb{L}_{d}[\phi]+\mathbb{N}_{d}[\phi]=-\mathbb{S}\left[V_{d}\right] .
$$

In the above, we have denoted the linear operator by

$$
\begin{aligned}
\mathbb{L}_{d}[\phi]= & \Delta \phi+\frac{1}{x_{1}} \frac{\partial \phi}{\partial x_{1}}-\frac{4 \bar{V}_{d}}{1+\left|V_{d}\right|^{2}} \nabla V_{d} \cdot \nabla \phi-\frac{2 \bar{\phi}}{1+\left|V_{d}\right|^{2}} \nabla V_{d} \cdot \nabla V_{d} \\
& +\frac{2 \bar{V}_{d}\left(V_{d} \bar{\phi}+\bar{V}_{d} \phi\right)}{\left(1+\left|V_{d}\right|^{2}\right)^{2}} \nabla V_{d} \cdot \nabla V_{d}-\frac{4 \bar{V}_{d}}{1+\left|V_{d}\right|^{2}} \gamma^{-2}\left[\frac{\lambda^{2}}{x_{1}^{2}}-\frac{\lambda^{2}}{d^{2}}\right] \frac{\partial V_{d}}{\partial x_{2}} \cdot \frac{\partial \phi}{\partial x_{2}} \\
& -\frac{2 \bar{\phi}}{1+\left|V_{d}\right|^{2}} \gamma^{-2}\left[\frac{\lambda^{2}}{x_{1}^{2}}-\frac{\lambda^{2}}{d^{2}}\right]\left(\frac{\partial V_{d}}{\partial x_{2}}\right)^{2}+\frac{2 \bar{V}_{d}\left(V_{d} \bar{\phi}+\bar{V}_{d} \phi\right)}{\left(1+\left|V_{d}\right|^{2}\right)^{2}} \gamma^{-2}\left[\frac{\lambda^{2}}{x_{1}^{2}}-\frac{\lambda^{2}}{d^{2}}\right]\left(\frac{\partial V_{d}}{\partial x_{2}}\right)^{2} \\
& +\gamma^{-2}\left[\frac{\lambda^{2}}{x_{1}^{2}}-\frac{\lambda^{2}}{d^{2}}\right] \frac{\partial^{2} \phi}{\partial x_{2}^{2}}+F^{\prime}\left(V_{d}\right) \phi
\end{aligned}
$$


The nonlinear operator is

$$
\begin{aligned}
\mathbb{N}_{d}[\phi]= & F\left(V_{d}+\phi\right)-F\left(V_{d}\right)-F^{\prime}\left(V_{d}\right) \phi+O\left((1+|\phi|)|\nabla \phi|^{2}\right) \\
& +\gamma^{-2}\left[\frac{\lambda^{2}}{x_{1}^{2}}-\frac{\lambda^{2}}{d^{2}}\right] O\left((1+|\phi|)|\nabla \phi|^{2}\right)+i \delta \gamma^{-1}|\log \epsilon| \epsilon \frac{\partial \phi}{\partial x_{2}} .
\end{aligned}
$$

In the above, we have used the definition of $F$ in (3.20). Note that in this region

$$
\frac{1}{x_{1}} \text { and }\left[\frac{\lambda^{2}}{x_{1}^{2}}-\frac{\lambda^{2}}{d^{2}}\right]
$$

are small terms. $\mathbb{L}_{d}$ is a small perturbation of $\mathbb{L}_{0}$ in (2.3).

In the outer region $\left\{z \in\left(B_{2}\left(d \vec{e}_{1}\right) \cup B_{2}\left(-d \vec{e}_{1}\right)\right)^{c}\right\}$, we have $u=V_{d} e^{i \psi}$. By simple computations we obtain

$$
\begin{aligned}
& \frac{\mathbb{S}\left[V_{d} e^{i \psi}\right]}{i V_{d} e^{i \psi}}=\Delta \psi+\frac{1}{x_{1}} \frac{\partial \psi}{\partial x_{1}}+2 \frac{1-\left|V_{d}\right|^{2}+\left|V_{d}\right|^{2}\left(e^{-2 \psi_{2}}-1\right)}{V_{d}\left(1+\left|V_{d}\right|^{2} e^{-2 \psi_{2}}\right)} \nabla V_{d} \cdot \nabla \psi \\
& +\frac{1}{i V_{d}} \frac{2\left|V_{d}\right|^{2} \bar{V}_{d}\left(e^{-2 \psi_{2}}-1\right)}{\left(1+\left|V_{d}\right|^{2}\right)\left(1+\left|V_{d}\right|^{2} e^{-2 \psi_{2}}\right)} \nabla V_{d} \cdot \nabla V_{d}-i\left(\frac{2\left|V_{d}\right|^{2}}{1+\left|V_{d}\right|^{2} e^{-2 \psi_{2}}}-1\right) \nabla \psi \cdot \nabla \psi \\
& -i \frac{4 \bar{V}_{d}}{1+\left|V_{d}\right|^{2} e^{-2 \psi_{2}}} \gamma^{-2}\left[\frac{\lambda^{2}}{x_{1}^{2}}-\frac{\lambda^{2}}{d^{2}}\right] \frac{\partial V_{d}}{\partial x_{2}} \cdot \frac{\partial \psi}{\partial x_{2}} \\
& +i \frac{1}{V_{d}} \frac{2 \bar{V}_{d}}{1+\left|V_{d}\right|^{2} e^{-2 \psi_{2}}} \gamma^{-2}\left[\frac{\lambda^{2}}{x_{1}^{2}}-\frac{\lambda^{2}}{d^{2}}\right] \frac{\partial V_{d}}{\partial x_{2}} \cdot \frac{\partial V_{d}}{\partial x_{2}} \\
& +\frac{2\left|V_{d}\right|^{2}}{1+\left|V_{d}\right|^{2} e^{-2 \psi_{2}}} \gamma^{-2}\left[\frac{\lambda^{2}}{x_{1}^{2}}-\frac{\lambda^{2}}{d^{2}}\right] \frac{\partial \psi}{\partial x_{2}} \cdot \frac{\partial \psi}{\partial x_{2}} \\
& -i \frac{2\left|V_{d}\right|^{2}\left(1-e^{-2 \psi_{2}}\right)}{\left(1+\left|V_{d}\right|^{2} e^{-2 \psi_{2}}\right)\left(1+\left|V_{d}\right|^{2}\right)}+\gamma^{-2}\left[\frac{\lambda^{2}}{x_{1}^{2}}-\frac{\lambda^{2}}{d^{2}}\right] \frac{\partial^{2} \psi}{\partial x_{2}^{2}} \\
& +i \delta \gamma^{-1}|\log \epsilon| \epsilon \frac{\partial \psi}{\partial x_{2}}+\frac{\mathbb{S}\left[V_{d}\right]}{i V_{d}} \text {. }
\end{aligned}
$$

We can also write the problem as an equation of $\psi=\psi_{1}+i \psi_{2}$

$$
\tilde{L}_{0}[\psi]+\tilde{N}_{1}[\psi]+\tilde{N}_{2}[\psi]=-\mathbb{S}\left[V_{d}\right] / i V_{d}
$$

with conditions in (5.4). In the above, we have denoted

$$
\begin{aligned}
& \tilde{L}_{0}[\psi]= \Delta \psi+\frac{1}{x_{1}} \frac{\partial \psi}{\partial x_{1}}+\frac{2\left(1-\left|V_{d}\right|^{2}\right)}{1+\left|V_{d}\right|^{2}} \nabla V_{d} \cdot \nabla \psi+\frac{4 i \bar{V}_{d}^{2} \psi_{2}}{\left(1+\left|V_{d}\right|^{2}\right)^{2}} \nabla V_{d} \cdot \nabla V_{d} \\
&+\frac{2\left(1-\left|V_{d}\right|^{2}\right)}{1+\left|V_{d}\right|^{2}} \gamma^{-2}\left[\frac{\lambda^{2}}{x_{1}^{2}}-\frac{\lambda^{2}}{d^{2}}\right] \frac{\partial V_{d}}{\partial x_{2}} \cdot \frac{\partial \psi}{\partial x_{2}}+\frac{4 i \bar{V}_{d}^{2} \psi_{2}}{\left(1+\left|V_{d}\right|^{2}\right)^{2}} \gamma^{-2}\left[\frac{\lambda^{2}}{x_{1}^{2}}-\frac{\lambda^{2}}{d^{2}}\right] \frac{\partial V_{d}}{\partial x_{2}} \cdot \frac{\partial V_{d}}{\partial x_{2}} \\
&-i \frac{4\left|V_{d}\right|^{2} \psi_{2}}{\left(1+\left|V_{d}\right|^{2}\right)^{2}}+\gamma^{-2}\left[\frac{\lambda^{2}}{x_{1}^{2}}-\frac{\lambda^{2}}{d^{2}}\right] \frac{\partial^{2} \psi}{\partial x_{2}^{2}}, \\
& \tilde{N}_{1}[\psi]= \frac{1}{V_{d}} \nabla \psi \cdot \nabla V_{d} O\left(\psi_{2}\right)+O\left(\left.|| V_{d}\right|^{2}-1|+| \psi_{2} \mid\right)|\nabla \psi \cdot \nabla \psi|+i O\left(\left|e^{-\psi_{2}}-1+\psi_{2}\right|\right), \\
& \tilde{N}_{2}[\psi]=i \delta \gamma^{-1}|\log \epsilon| \epsilon \frac{\partial \psi}{\partial x_{2}} .
\end{aligned}
$$


Recall that $\psi=\psi_{1}+i \psi_{2}$. Then setting $z=d \vec{e}_{1}+y$, we have for $z \in \mathbb{R}_{+}^{2}$

$$
\begin{gathered}
\tilde{L}_{0}[\psi]=\left[\begin{array}{c}
\Delta \psi_{1}+\frac{1}{x_{1}} \frac{\partial \psi_{1}}{\partial x_{1}}+\gamma^{-2}\left(\frac{\lambda^{2}}{x_{1}^{2}}-\frac{\lambda^{2}}{d^{2}}\right) \frac{\partial^{2} \psi_{1}}{\partial x_{2}^{2}}+O\left(e^{-|y|}\right)|\nabla \psi| \\
\Delta \psi_{2}+\frac{1}{x_{1}} \frac{\partial \psi_{2}}{\partial x_{1}}+\gamma^{-2}\left(\frac{\lambda^{2}}{x_{1}^{2}}-\frac{\lambda^{2}}{d^{2}}\right) \frac{\partial^{2} \psi_{2}}{\partial x_{2}^{2}}-\frac{4\left|V_{d}\right|^{2}}{\left(1+\left|V_{d}\right|^{2}\right)^{2}} \psi_{2}+O\left(e^{-|y|}\right) \nabla \psi_{2}
\end{array}\right], \\
\tilde{N}_{1}[\psi]=\left[\begin{array}{c}
O\left(e^{-|y|}|\nabla \psi \cdot \nabla \psi|+\left|\psi_{2}\right|^{2} \frac{1}{(1+|y|)^{2}}+\left|\psi_{2}\right| \frac{1}{1+|y|}|\nabla \psi|\right) \\
O\left(e^{-|y|}|\nabla \psi \cdot \nabla \psi|+\left|\psi_{2}\right||\nabla \psi \cdot \nabla \psi|+\left|\psi_{2}\right|^{2}\right)
\end{array}\right], \\
\tilde{N}_{2}[\psi]=\left[\begin{array}{c}
O\left(\epsilon\left|\frac{\partial \psi_{2}}{\partial y_{2}}\right|\right) \\
O\left(\epsilon\left|\frac{\partial \psi_{1}}{\partial y_{2}}\right|\right)
\end{array}\right] .
\end{gathered}
$$

Note that

$$
\Delta+\frac{1}{x_{1}} \frac{\partial}{\partial x_{1}}+\gamma^{-2}\left[\frac{\lambda^{2}}{x_{1}^{2}}-\frac{\lambda^{2}}{d^{2}}\right] \frac{\partial^{2}}{\partial x_{2}^{2}}=\frac{\partial^{2}}{\partial x_{1}^{2}}+\frac{1}{\gamma^{2}} \frac{\partial^{2}}{\partial x_{2}^{2}}+\frac{1}{x_{1}} \frac{\partial}{\partial x_{1}}+\frac{\lambda^{2}}{\gamma^{2}} \frac{1}{x_{1}^{2}} \frac{\partial^{2}}{\partial x_{2}^{2}} .
$$

Let us remark that the explicit form of all the linear and nonlinear terms will be very useful for later analysis in resolution theory.

Define

$$
\mathbb{E}_{d}=-\mathbb{S}\left[V_{d}\right], \quad \tilde{\mathbb{E}}_{d}=-\mathbb{S}\left[V_{d}\right] / i V_{d}
$$

Based on the form of the errors, we need to use suitable norms. Let us fix two positive numbers

$$
p>13, \quad 0<\varrho<1 .
$$

Recall that $\phi=i V_{d} \psi, \psi=\psi_{1}+i \psi_{2}, \ell_{1}=\left|z+d \vec{e}_{1}\right|$ and $\ell_{2}=\left|z+d \vec{e}_{1}\right|$. We define

$$
\begin{gathered}
\|h\|_{* *}=\left\|i V_{d} h\right\|_{L^{p}\left(\mathfrak{S}_{1}\right)}+\sum_{j=1}^{2}\left[\left\|\ell_{j}^{2+\varrho} h_{1}\right\|_{L^{\infty}\left(\mathfrak{S}_{2}\right)}+\left\|\ell_{j}^{1+\varrho} h_{2}\right\|_{L^{\infty}\left(\mathfrak{S}_{2}\right)}\right] \\
\|\psi\|_{*}=\|\phi\|_{W^{2, p}\left(\mathfrak{S}_{1}\right)}+\sum_{j=1}^{2}\left[\left\|\ell_{j}^{\varrho} \psi_{1}\right\|_{L^{\infty}\left(\mathfrak{S}_{2}\right)}+\left\|\ell_{j}^{1+\varrho} \nabla \psi_{1}\right\|_{L^{\infty}\left(\mathfrak{S}_{2}\right)}\right] \\
+\left[\left\|\ell_{j}^{1+\varrho} \psi_{2}\right\|_{L^{\infty}\left(\mathfrak{S}_{2}\right)}+\left\|\ell_{j}^{2+\varrho} \nabla \psi_{2}\right\|_{L^{\infty}\left(\mathfrak{S}_{2}\right)}\right] .
\end{gathered}
$$

In the above,

$$
\begin{array}{r}
\mathfrak{S}_{1}=\left\{z \in \mathfrak{S}:\left|z-d \vec{e}_{1}\right|<3 \text { or }\left|z+d \vec{e}_{2}\right|<3\right\}, \\
\mathfrak{S}_{2}=\left\{z \in \mathfrak{S}:\left|z-d \vec{e}_{1}\right|>2 \text { and }\left|z+d \vec{e}_{2}\right|>2\right\} .
\end{array}
$$

We remark that we use the norm $L_{l o c}^{p}\left(\right.$ or $W_{l o c}^{2, p}$ ) in the inner part due to the fact that the error term contains terms like $\epsilon \log \ell_{1}$ which is not $L^{\infty}$-bounded.

Using the norms defined above, we can have the following error estimates. For the proof, the reader can refer to Lemma 4.1.

Lemma 5.1. It holds that for $z \in \mathfrak{S}_{2}$

$$
\begin{gathered}
\left|\operatorname{Re}\left(\tilde{\mathbb{E}}_{d}\right)\right| \leq \frac{C \epsilon^{1-\varrho}}{\left(1+\ell_{1}\right)^{3}}+\frac{C \epsilon^{1-\varrho}}{\left(1+\ell_{2}\right)^{3}}, \\
\left|\operatorname{Im}\left(\tilde{\mathbb{E}}_{d}\right)\right| \leq \frac{C \epsilon^{1-\varrho}}{\left(1+\ell_{1}\right)^{1+\varrho}}+\frac{C \epsilon^{1-\varrho}}{\left(1+\ell_{2}\right)^{1+\varrho}},
\end{gathered}
$$


and also

$$
\left\|\mathbb{E}_{d}\right\|_{L^{p}\left(\mathfrak{S}_{1}\right)} \leq C \epsilon \log \frac{1}{\epsilon}
$$

where $\varrho \in(0,1)$ is a constant. As a consequence, there holds

$$
\left\|\tilde{\mathbb{E}}_{d}\right\|_{* *} \leq C \epsilon^{1-\varrho} .
$$

\section{Projected Linear and Nonlinear Problems}

Let $L$ be a linear operator in the form

$$
\mathcal{L}=\mathbb{L}_{d} \quad \text { in } \quad \mathfrak{S}_{1}, \quad \mathcal{L}=\tilde{L}_{0} \quad \text { in } \quad \mathfrak{S}_{2},
$$

and the co-kernel

$$
Z_{d}:=\frac{\partial V_{d}}{\partial d}\left[\tilde{\eta}\left(\frac{\ell_{1}}{R}\right)+\tilde{\eta}\left(\frac{\ell_{2}}{R}\right)\right]
$$

where $\tilde{\eta}$ is defined at (5.2) before. Then $Z_{d}$ satisfies the symmetry (3.16). For $\psi=\psi_{1}+i \psi_{2}$, we require the conditions

$$
\begin{array}{rlrl}
\psi_{1}\left(x_{1}, x_{2}\right)=\psi_{1}\left(-x_{1}, x_{2}\right), & \psi_{1}\left(x_{1}, x_{2}\right)=-\psi_{1}\left(x_{1},-x_{2}\right), \\
\psi_{2}\left(x_{1}, x_{2}\right)=\psi_{2}\left(-x_{1}, x_{2}\right), & \psi_{2}\left(x_{1}, x_{2}\right)=\psi_{2}\left(x_{1},-x_{2}\right), \\
\frac{\partial \psi_{1}}{\partial x_{1}}\left(0, x_{2}\right)=0, & & \frac{\partial \psi_{2}}{\partial x_{1}}\left(0, x_{2}\right)=0, \\
\psi_{1}\left(x_{1},-\lambda \pi / \gamma\right)=\psi_{1}\left(x_{1}, \lambda \pi / \gamma\right)=0, & & \frac{\partial \psi_{1}}{\partial x_{2}}\left(x_{1},-\lambda \pi / \gamma\right)=\frac{\partial \psi_{1}}{\partial x_{2}}\left(x_{1}, \lambda \pi / \gamma\right), \\
\frac{\partial \psi_{2}}{\partial x_{2}}\left(x_{1},-\lambda \pi / \gamma\right)=0, & & \frac{\partial \psi_{2}}{\partial x_{2}}\left(x_{1}, \lambda \pi / \gamma\right)=0, \\
\psi_{2}\left(x_{1},-\lambda \pi / \gamma\right)=\psi_{2}\left(x_{1}, \lambda \pi / \gamma\right) . &
\end{array}
$$

As the first step of finite dimensional reduction, we need to consider the following linear problem

$$
\left\{\begin{array}{l}
\mathcal{L}(\psi)=h \text { in } \mathfrak{S}, \quad \psi \text { satisfies the conditions in }(6.2), \\
\operatorname{Re}\left(\int_{\mathfrak{S}} \bar{\phi} Z_{d} \mathrm{~d} x\right)=0 \quad \text { for } \phi=i V_{d} \psi \text { in } B_{1}\left(d \vec{e}_{1}\right) \cup B_{1}\left(-d \vec{e}_{1}\right) .
\end{array}\right.
$$

We have the following a priori estimates.

Lemma 6.1. There exists a constant $C$, depending on @ only such that for all $\epsilon$ sufficiently small, $d \sim \frac{1}{\epsilon}$, and any solution of (6.3), it holds

$$
\|\psi\|_{*} \leq C\|h\|_{* *} .
$$

Proof: The proof is similar as in Lemma 5.1 in [34]. Suppose that there exists a sequence of $\epsilon=\epsilon_{n} \rightarrow 0$, functions $\psi^{n}, h_{n}$ which satisfy (6.3) with

$$
\left\|\psi^{n}\right\|_{*}=1, \quad\left\|h_{n}\right\|_{* *}=o(1)
$$

We will derive a contradiction by careful analysis of the estimates.

We derive inner estimates first. We have the symmetries and boundary conditions for $\psi_{1}$ and $\psi_{2}$ in (6.2). Whence we may just need to consider the region

$$
\Sigma_{+}=\left\{x_{1}>0,0<x_{2}<\lambda \pi / \gamma\right\} .
$$

Then we have

$$
\operatorname{Re}\left(\int_{\mathbb{R}^{2}} \bar{\phi}_{n} Z_{d}\right)=2 \operatorname{Re}\left(\int_{\Sigma_{+}} \bar{\phi}_{n} Z_{d}\right)=0
$$


Let $z \in \Sigma_{+}, z=d \vec{e}_{1}+y$ and $\tilde{\phi}_{n}(y)=\phi_{n}(z)$. Then as $n \rightarrow+\infty$,

$$
V_{d}=w^{+}(y) e^{-i \theta_{-d \vec{e}_{1}}}\left(1+O\left(e^{-d / 2}\right)\right)=-w^{+}(y)+o(1) .
$$

Since $\left\|\psi^{n}\right\|_{*}=1$, we may take a limit so that $\tilde{\phi}_{n} \rightarrow \phi_{0}$ in $\mathbb{R}_{l o c}^{2}$, where $\phi_{0}$ satisfies

$$
\mathbb{L}_{0}\left[\phi_{0}\right]=0
$$

where $\mathbb{L}_{0}$ is defined by (2.4). Observe that $\phi_{0}$ satisfies the decay estimate (2.5) because of our assumption on $\psi^{n}$. By Lemma 2.2, we have

$$
\phi_{0}=c_{1} \frac{\partial w}{\partial y_{1}}+c_{2} \frac{\partial w}{\partial y_{2}} .
$$

Observe that $\phi_{0}$ inherits the symmetries of $\phi$ and hence $\phi_{0}=\overline{\phi_{0}(\bar{z})}$. (The other symmetry is not preserved under the transformation $z=d \vec{e}_{1}+y$.) But certainly $\frac{\partial w}{\partial y_{2}}$ does not enjoy the above symmetry. Hence $\phi_{0}=c_{1} \frac{\partial w}{\partial y_{1}}$. On the other hand, taking a limit of the orthogonality condition

$$
\operatorname{Re}\left(\int_{\Sigma_{+}} \bar{\phi}_{n} Z_{d}\right)=0
$$

we obtain

$$
\operatorname{Re}\left(\int_{\mathbb{R}^{2}} \overline{\phi_{0}} \frac{\partial w}{\partial y_{1}}\right)=0
$$

This implies that $c_{1}=0$ and hence we have

$$
\phi_{n} \rightarrow 0 \text { in } \mathbb{R}_{l o c}^{2}
$$

which implies that for any fixed $R>0$,

$$
\sum_{j=1}^{2}\left(\left\|\phi_{1}\right\|_{L^{p}\left(\ell_{j}<R\right)}+\left\|\phi_{2}\right\|_{L^{p}\left(\ell_{j}<R\right)}+\left\|\nabla \phi_{1}\right\|_{L^{p}\left(\ell_{j}<R\right)}+\left\|\nabla \phi_{2}\right\|_{L^{p}\left(\ell_{j}<R\right)}\right)=o(1) .
$$

We use the $L^{p}$-estimates in the inner part $\left\{\left|z-d \vec{e}_{1}\right|<R\right\}$. By choosing $p$ large we obtain the embedding $W_{l o c}^{2, p}$ into $C_{l o c}^{1, \alpha}$ for any $\alpha \in(0,1)$.

Next we shall derive outer estimates. Again, by using the symmetries and boundary conditions for $\psi_{1}$ and $\psi_{2}$ in (6.2). We just need to consider the region

$$
\Sigma=\left\{x_{1} \in \mathbb{R}, 0<x_{2}<\lambda \pi / \gamma\right\} .
$$

Let $\tilde{\eta}$ be a cut-off function such that $\tilde{\eta}(s)=1$ for $s \leq 1$ and $\tilde{\eta}(s)=0$ for $s>2$. We consider the new function

$$
\tilde{\psi}=\psi \chi(z), \text { where } \chi(z)=1-\tilde{\eta}\left(\frac{\ell_{1}}{4}\right)-\tilde{\eta}\left(\frac{\ell_{2}}{4}\right) .
$$

Using the explicit forms of $\tilde{L}_{0}$ in (5.10), the first equation becomes

$$
\begin{aligned}
& \frac{\partial^{2} \tilde{\psi}_{1}}{\partial x_{1}^{2}}+\frac{1}{x_{1}} \frac{\partial \tilde{\psi}_{1}}{\partial x_{1}}+\frac{1}{\gamma^{2}} \frac{\partial^{2} \tilde{\psi}_{1}}{\partial x_{2}^{2}}+\frac{\lambda^{2}}{\gamma^{2}} \frac{1}{x_{1}^{2}} \frac{\partial^{2} \tilde{\psi}_{1}}{\partial x_{2}^{2}} \\
& =O\left(e^{-|y|}\right)|\nabla \psi|+O\left(\nabla \chi \nabla \psi_{1}\right)+O\left(\psi_{1} \Delta \chi\right)+h_{1} \chi .
\end{aligned}
$$

On the region $\Sigma \backslash\left(B_{4}\left(\vec{e}_{1}\right) \cup B_{4}\left(-d \vec{e}_{1}\right)\right)$, we have the conditions in (6.2). Moreover we have

$$
|\nabla \chi \cdot \nabla \psi|=o(1)\left(\ell_{1}^{2}+\ell_{2}^{2}\right)^{-\frac{\sigma+2}{2}} .
$$

For the outer part estimates, we use the following new barrier function

$$
B(z):=B_{1}(z)+B_{2}(z),
$$

where by the notation

$$
\tilde{\ell}_{1}=\sqrt{\left(x_{1}-d\right)^{2}+k^{2} x_{2}^{2}}, \quad \tilde{\ell}_{2}=\sqrt{\left(x_{1}+d\right)^{2}+k^{2} x_{2}^{2}},
$$


we have denoted

$$
B_{1}(z)=\left(\eta\left(\epsilon \ell_{1}\right)+\eta\left(\epsilon \ell_{2}\right)\right)\left(\tilde{\ell}_{1}^{\beta} x_{2}^{\nu}+\tilde{\ell}_{2}^{\beta} x_{2}^{\nu}\right), \quad B_{2}(z)=x_{1}^{-\varrho}\left(\sin \left(\tau \epsilon x_{2}\right)\right)^{\nu} .
$$

In the above $\beta+\nu=-\varrho, 0<\varrho<\nu<1 . \eta$ is a smooth cut-off function with properties $\eta(t)=1$ for $|t|<c_{\varrho}$, where $c_{\varrho}$ is small, and $\eta(t)=0$ for $|t|>2 c_{\varrho}$. Note that $c_{\varrho}, \tau$ and $k$ are constants, which will be determined in the sequel.

Now, we do the computations for $B_{1}$

$$
\frac{\partial^{2} B_{1}}{\partial x_{1}^{2}}+\frac{1}{k^{2}} \frac{\partial^{2} B_{1}}{\partial x_{2}^{2}} \leq-C_{1}\left(\tilde{\ell}_{1}^{-2-\varrho}+\tilde{\ell}_{2}^{-2-\varrho}\right)
$$

where $C_{1}$ depends only on $\beta$ and $\nu$. On the other hand, there holds

$$
\frac{1}{x_{1}} \frac{\partial B_{1}}{\partial x_{1}} \leq C \frac{x_{2}^{\nu}}{x_{1}}\left[\tilde{\ell}_{1}^{\beta-2}\left(x_{1}-d\right)+\tilde{\ell}_{2}^{\beta-2}\left(x_{1}+d\right)\right] .
$$

Thus for $\left|x_{1}-d\right|<c_{\varrho} d$ or $\left|x_{1}+d\right|<c_{\varrho} d$, where $c_{\varrho}$ is small, we have

$$
\frac{1}{x_{1}} \frac{\partial B_{1}}{\partial x_{1}} \leq C_{2} c_{\varrho}\left(\tilde{\ell}_{1}^{-2-\varrho}+\tilde{\ell}_{2}^{-2-\varrho}\right)
$$

where $C_{2}$ depends only on $\beta$ and $\nu$. Furthermore, we can choose $k$ such that there exists a positive constant $C_{3}$ depends only on $\beta, \nu, \hat{\lambda}$ and $\gamma$, for $\left|x_{1}-d\right|<c_{\varrho} d$ or $\left|x_{1}+d\right|<c_{\varrho} d$ there holds

$$
\begin{aligned}
& \left(\frac{\lambda^{2}}{\gamma^{2}} \frac{1}{x_{1}^{2}}+\frac{1}{\gamma^{2}}-\frac{1}{k^{2}}\right) \frac{\partial^{2} B_{1}}{\partial x_{2}^{2}} \\
& =\left(\frac{\hat{\lambda}^{2}}{\gamma^{2}} \frac{1}{\epsilon^{2} x_{1}^{2}}+\frac{1}{\gamma^{2}}-\frac{1}{k^{2}}\right)\left[\beta(2 \nu+1)+\beta(\beta-2) \tilde{\ell}^{-2} x_{2}^{2}+\nu(\nu-1) \tilde{\ell}_{1}^{2} x_{2}^{-2}\right] \tilde{\ell}_{1}^{-2+\beta}\left(\gamma x_{2}\right)^{\nu} \\
& \quad+\left(\frac{\hat{\lambda}^{2}}{\gamma^{2}} \frac{1}{\epsilon^{2} x_{1}^{2}}+\frac{1}{\gamma^{2}}-\frac{1}{k^{2}}\right)\left[\beta(2 \nu+1)+\beta(\beta-2) \tilde{\ell}^{-2} x_{2}^{2}+\nu(\nu-1) \tilde{\ell}_{2}^{2} x_{2}^{-2}\right] \tilde{\ell}_{2}^{-2+\beta}\left(\gamma x_{2}\right)^{\nu} \\
& \leq C_{3} c_{\varrho}\left(\tilde{\ell}_{1}^{-2-\varrho}+\tilde{\ell}_{2}^{-2-\varrho}\right) .
\end{aligned}
$$

By choosing $c_{\varrho}$ small, we obtain

$$
\frac{\partial^{2} B_{1}}{\partial x_{1}^{2}}+\frac{1}{x_{1}} \frac{\partial B_{1}}{\partial x_{1}}+\frac{1}{\gamma^{2}} \frac{\partial^{2} B_{1}}{\partial x_{2}^{2}}+\frac{\lambda^{2}}{\gamma^{2}} \frac{1}{x_{1}^{2}} \frac{\partial^{2} B_{1}}{\partial x_{2}^{2}} \leq C\left(\tilde{\ell}_{1}^{-2-\varrho}+\tilde{\ell}_{2}^{-2-\varrho}\right) .
$$

Note that we only do the computations by ignoring the cut-off function in the expressions of $B_{1}$.

By trivial computations, we obtain that

$$
\begin{aligned}
& \frac{\partial^{2} B_{2}}{\partial x_{1}^{2}}+\frac{1}{x_{1}} \frac{\partial B_{2}}{\partial x_{1}}+\frac{1}{\gamma^{2}} \frac{\partial^{2} B_{2}}{\partial x_{2}^{2}}+\frac{\lambda^{2}}{\gamma^{2}} \frac{1}{x_{1}^{2}} \frac{\partial^{2} B_{2}}{\partial x_{2}^{2}} \\
& =\varrho^{2} x_{1}^{-2-\varrho}\left(\sin \left(\tau \epsilon x_{2}\right)\right)^{\nu} \\
& \quad-\nu^{2} \gamma^{-2} \tau^{2} \epsilon^{2} x_{1}^{-\varrho}\left(\sin \left(\tau \epsilon x_{2}\right)\right)^{\nu}+\nu(\nu-1) \gamma^{-2} \tau^{2} \epsilon^{2} x_{1}^{-\varrho}\left(\sin \left(\tau \epsilon x_{2}\right)\right)^{\nu-2} \\
& \quad-\nu^{2} \frac{\hat{\lambda}^{2}}{\gamma^{2}} \tau^{2} x_{1}^{-2-\varrho}\left(\sin \left(\tau \epsilon x_{2}\right)\right)^{\nu}+\nu(\nu-1) \frac{\hat{\lambda}^{2}}{\gamma^{2}} \tau^{2} x_{1}^{-2-\varrho}\left(\sin \left(\tau \epsilon x_{2}\right)\right)^{\nu-2}
\end{aligned}
$$

By choosing $\tau$ small enough, we have

$$
0<\sin \left(\tau \epsilon x_{2}\right)<1 / 2 \quad \text { for } 0<x_{2}<\lambda \pi / \gamma \text {. }
$$

If $x_{1}-d<-c_{\varrho} d$, there holds

$$
x_{1}^{2}<\left(\frac{1-c_{\varrho}}{c_{\varrho}}\right)^{2}\left(x_{1}-d\right)^{2} .
$$

On the other hand, $x_{1}-d>c_{\varrho} d$, there holds

$$
x_{1}^{2}<\left(\frac{1+c_{\varrho}}{c_{\varrho}}\right)^{2}\left(x_{1}-d\right)^{2} .
$$


We finally have

$$
\frac{\partial^{2} B}{\partial x_{1}^{2}}+\frac{1}{x_{1}} \frac{\partial B}{\partial x_{1}}+\frac{1}{\gamma^{2}} \frac{\partial^{2} B}{\partial x_{2}^{2}}+\frac{\lambda^{2}}{\gamma^{2}} \frac{1}{x_{1}^{2}} \frac{\partial^{2} B}{\partial x_{2}^{2}} \leq-C\left(\ell_{1}^{-2-\varrho}+\ell_{2}^{-2-\varrho}\right) .
$$

By comparison principle on the set $\Sigma \backslash\left(B_{4}\left(d \vec{e}_{1}\right) \cup B_{4}\left(-d \vec{e}_{1}\right)\right)$, we get that

$$
\left|\tilde{\psi}_{1}\right| \leq C B\left(\|h\|_{* *}+o(1)\right), \quad \forall z \in \Sigma \backslash\left(B_{4}\left(d \vec{e}_{1}\right) \cup B_{4}\left(-d \vec{e}_{1}\right)\right) .
$$

Elliptic estimates then give

$$
\sum_{j=1}^{2}\left\|\ell_{j}^{1+\sigma}\left|\nabla \tilde{\psi}_{1}\right|\right\|_{L^{\infty}\left(\ell_{j}>4\right)} \leq C\left(\|h\|_{* *}+o(1)\right) .
$$

To estimate $\psi_{2}$, we perform the same cut-off and now the second equation becomes

$$
\begin{aligned}
& \frac{\partial^{2} \tilde{\psi}_{2}}{\partial x_{1}^{2}}+\frac{1}{x_{1}} \frac{\partial \tilde{\psi}_{2}}{\partial x_{1}}+\frac{1}{\gamma^{2}} \frac{\partial^{2} \tilde{\psi}_{2}}{\partial x_{2}^{2}}+\frac{\lambda^{2}}{\gamma^{2}} \frac{1}{x_{1}^{2}} \frac{\partial^{2} \tilde{\psi}_{2}}{\partial x_{2}^{2}}-\frac{4\left|V_{d}\right|^{2}}{\left(1+\left|V_{d}\right|^{2}\right)^{2}} \tilde{\psi}_{2} \\
& =O\left(\frac{1}{1+|y|}\right) \nabla \psi_{1}+O\left(e^{-|y|}\right) \nabla \psi_{2}+O\left(\nabla \chi \nabla \psi_{2}\right)+O\left(\Delta \psi_{2}\right)+h_{2} \chi .
\end{aligned}
$$

Note that we also have the conditions in (6.2). Since for $z \in \Sigma \backslash\left(B_{4}\left(d \vec{e}_{1}\right) \cup B_{4}\left(-d \vec{e}_{1}\right)\right)$, there holds

$$
\frac{4\left|V_{d}\right|^{2}}{\left(1+\left|V_{d}\right|^{2}\right)} \geq \frac{1}{4}
$$

by standard elliptic estimates we have

$$
\begin{aligned}
\left\|\psi_{2}\right\|_{L^{\infty}\left(\ell_{j}>4\right)} & \leq C\left(\left\|\psi_{2}\right\|_{L^{\infty}\left(\ell_{j}=4\right)}\right)\left(1+\|\psi\|_{*}\right)\|h\|_{* *}\left(1+\ell_{1}+\ell_{2}\right)^{-1-\varrho} \\
\left|\nabla \psi_{2}\right| & \leq C\left(\left\|\psi_{2}\right\|_{L^{\infty}\left(\ell_{j}=R\right)}\right)\left(1+\|\psi\|_{*}\right)\|h\|_{* *}\left(1+\ell_{1}+\ell_{2}\right)^{-2-\varrho}
\end{aligned}
$$

Combining both inner and outer estimates in (6.5), (6.15)-(6.16) and (6.18), we obtain that $\|\psi\|_{*}=o(1)$, which is a contradiction.

By contraction mapping theorem, we conclude

Proposition 6.2. There exists a constant $C$, depending on $\nu, \varrho$ only such that for all $\epsilon$ sufficiently small, $d$ large, the following holds: there exists a unique solution $\left(\phi_{\epsilon, d}, \mathcal{C}_{\epsilon}(d)\right)$ to

$$
\mathbb{S}\left[V_{d}+\phi_{\epsilon, d}\right]=\mathcal{C}_{\epsilon}(d) Z_{d}
$$

with conditions in (5.3) by the relation $\phi_{\epsilon, d}=i V_{d} \psi$ and $\phi_{\epsilon, d}$ satisfies

$$
\left\|\phi_{\epsilon, d}\right\|_{*} \leq C \epsilon^{1-\varrho} \text {. }
$$

Furthermore, $\phi_{\epsilon, d}$ is continuous in the parameter $d$.

Here, we do not give the proof to the last proposition. The reader can refer to the arguments in [34] for details.

\section{Reduced Problem and the Proof of Theorem 1.2}

We now solve the reduced problem. From Proposition 6.2, we deduce the existence of a solution $(\phi, \mathcal{C})=\left(\phi_{\epsilon, d}, \mathcal{C}_{\epsilon}(d)\right)$ to

$$
\mathbb{S}\left[V_{d}+\phi_{\epsilon, d}\right]=\mathbb{L}_{d}\left[\phi_{\epsilon, d}\right]+\mathbb{N}_{d}\left[\phi_{\epsilon, d}\right]+\mathbb{S}\left[V_{d}\right]=\mathcal{C}_{\epsilon}(d) Z_{d} .
$$

Multiplying (7.1) by $\frac{1}{\left(1+\left|V_{d}\right|^{2}\right)^{2}} \overline{Z_{d}}$ and integrating, we obtain

$$
\begin{aligned}
\mathcal{C}_{\epsilon} \operatorname{Re}\left(\int_{\mathbb{R}^{2}} \frac{1}{\left(1+\left|V_{d}\right|^{2}\right)^{2}} \overline{Z_{d}} Z_{d}\right)= & \operatorname{Re}\left(\int_{\mathbb{R}^{2}} \frac{1}{\left(1+\left|V_{d}\right|^{2}\right)^{2}} \overline{Z_{d}} \mathbb{S}\left[V_{d}\right]\right)+\operatorname{Re}\left(\int_{\mathbb{R}^{2}} \frac{1}{\left(1+\left|V_{d}\right|^{2}\right)^{2}} \overline{Z_{d}} \mathbb{L}_{d}\left[\phi_{\epsilon, d}\right]\right) \\
& +\operatorname{Re}\left(\int_{\mathbb{R}^{2}} \frac{1}{\left(1+\left|V_{d}\right|^{2}\right)^{2}} \overline{Z_{d}} \mathbb{N}_{d}\left[\phi_{\epsilon, d}\right]\right) .
\end{aligned}
$$


Using Proposition 6.2 and the expression in (5.8), we deduce that

$$
\operatorname{Re}\left(\int_{\mathbb{R}^{2}} \overline{Z_{d}} \mathbb{N}_{d}\left[\phi_{\epsilon, d}\right]\right)=o(\epsilon) .
$$

On the other hand, integration by parts, we have

$$
\operatorname{Re}\left(\int_{\mathbb{R}^{2}} \frac{1}{\left(1+\left|V_{d}\right|^{2}\right)^{2}} \overline{Z_{d}} \mathbb{L}_{d}\left[\phi_{\epsilon, d}\right]\right)=\operatorname{Re}\left(\int_{\mathbb{R}^{2}} \frac{1}{\left(1+\left|V_{d}\right|^{2}\right)^{2}} \overline{\phi_{\epsilon, d}} \mathbb{L}_{d}\left[Z_{d}\right]\right) .
$$

Let us observe that

and thus by Proposition 6.2

$$
\frac{\partial}{\partial d} \mathbb{S}_{0}\left[V_{d}\right]=\mathbb{L}_{d}\left[\frac{\partial V_{d}}{\partial d}\right]=\mathbb{L}_{d}\left[Z_{d}\right]=O(\epsilon)
$$

$$
\operatorname{Re}\left(\int_{\mathbb{R}^{2}} \frac{1}{\left(1+\left|V_{d}\right|^{2}\right)^{2}} \overline{\phi_{\epsilon, d}} \mathbb{L}_{d}\left[Z_{d}\right]\right)=o(\epsilon)
$$

As the strategy in standard reduction method, we are left to estimate the following integral

$$
\operatorname{Re}\left(\int_{\mathfrak{S}_{+}} \frac{1}{\left(1+\left|V_{d}\right|^{2}\right)^{2}} \overline{Z_{d}} \mathbb{S}\left[V_{d}\right]\right)=\operatorname{Re}\left[\int_{\mathfrak{S}_{+}} \frac{1}{\left(1+\left|V_{d}\right|^{2}\right)^{2}} \overline{Z_{d}} \sum_{i=0}^{3}\left(\Omega_{1 i}+\Omega_{2 i}\right) e^{i \tilde{\varphi}}\right]
$$

The expressions of these error terms are given in Sections 4.1 and 4.3. See (4.7), (4.8), (4.14), (4.18), (4.47), (4.48) and (4.49). We will estimate the above integrals in the sequel.

On $\mathfrak{S}_{+}$, recall that $z=d \vec{e}_{1}+y$. For the simplification of notation, we denote $\theta=\theta_{d \vec{e}_{1}}$ in the translated variables. By the properties of $\rho$, we have

$$
\begin{aligned}
v_{0} & =w(y) e^{-i \theta_{-d \vec{e}_{1}}}\left[1+O\left(e^{-d / 2-|y| / 2}\right)\right] \\
\left|v_{0}\right| & =\tilde{\rho}=\rho(|y|)\left[1+O\left(e^{-d / 2-|y| / 2}\right)\right] \\
\overline{Z_{d}} & =\tilde{\eta}(|y| / R)\left[-\frac{\partial \tilde{\rho}}{\partial y_{1}}-i \tilde{\rho} \frac{\partial \theta}{\partial y_{1}}\right] e^{-i \tilde{\varphi}}+O(\epsilon) .
\end{aligned}
$$

These asymptotic behaviors will be helpful to simplify the computations in the sequel. We first deal with

$$
\begin{aligned}
\operatorname{Re} & {\left[\int_{\mathfrak{S}_{+}} \frac{1}{\left(1+\left|V_{d}\right|^{2}\right)^{2}} \overline{Z_{d}}\left(\Omega_{10}+\Omega_{12}+\Omega_{20}+\Omega_{22}\right) e^{i \tilde{\varphi}}\right] } \\
= & -\int_{\mathfrak{S}_{+}} \frac{1}{\left(1+\rho^{2}\right)^{2}}\left(\frac{1}{d+y_{1}} \frac{\partial \tilde{\rho}}{\partial y_{1}}+\frac{\tilde{\rho}\left(\tilde{\rho}^{2}-1\right)}{\tilde{\rho}^{2}+1}\left(2 \nabla \varphi_{s} \nabla \theta+\left|\nabla \varphi_{s}\right|^{2}\right)\right) \frac{\partial \tilde{\rho}}{\partial y_{1}} \\
& -\int_{\mathfrak{S}_{+}} \frac{1}{\left(1+\rho^{2}\right)^{2}} \frac{2 \tilde{\rho}\left(1-\tilde{\rho}^{2}\right)}{1+\tilde{\rho}^{2}} \nabla \tilde{\rho} \cdot \nabla \varphi_{s} \frac{\partial \theta}{\partial y_{1}}+O(\epsilon) .
\end{aligned}
$$

where

$$
\int_{\mathfrak{S}_{+}} \frac{1}{\left(1+\rho^{2}\right)^{2}} \frac{1}{d+y_{1}}\left(\frac{\partial \tilde{\rho}}{\partial y_{1}}\right)^{2}=O(\epsilon)
$$

Let us notice that

Hence

$$
\nabla \varphi_{s}=-\frac{1}{2 d} \log d \nabla y_{2}+O(\epsilon \log |y|)
$$

$$
\begin{aligned}
& -\int_{\mathfrak{S}_{+}} \frac{1}{\left(1+\rho^{2}\right)^{2}}\left[\frac{\tilde{\rho}\left(\tilde{\rho}^{2}-1\right)}{\tilde{\rho}^{2}+1}\left(2 \nabla \varphi_{s} \cdot \nabla \theta+\left|\nabla \varphi_{s}\right|^{2}\right) \frac{\partial \tilde{\rho}}{\partial y_{1}}+\frac{2 \tilde{\rho}\left(1-\tilde{\rho}^{2}\right)}{1+\tilde{\rho}^{2}} \nabla \tilde{\rho} \cdot \nabla \varphi_{s} \frac{\partial \theta}{\partial y_{1}}\right]+O(\epsilon) \\
& =\frac{-1}{d \gamma^{2}} \log d \int_{\mathfrak{S}_{+}} \frac{1-\rho^{2}}{\left(\rho^{2}+1\right)^{3}} \frac{\rho \rho^{\prime}}{|y|} d y+O(\epsilon)
\end{aligned}
$$




$$
=\frac{-\pi}{4 d \gamma^{2}} \log d+O(\epsilon)
$$

Similarly, we can estimate the next two terms in (7.2)

$$
\begin{aligned}
& \operatorname{Re}\left[\int_{\mathfrak{S}_{+}} \frac{1}{\left(1+\left|V_{d}\right|^{2}\right)^{2}} \overline{Z_{d}}\left(\Omega_{11}+\Omega_{21}\right) e^{i \tilde{\varphi}}\right] \\
&=-\gamma^{-2} \int_{\mathfrak{S}_{+}} \frac{1}{\left(1+\rho^{2}\right)^{2}}\left(\frac{\partial^{2} \tilde{\rho}}{\partial x_{2}^{2}}-\frac{2 \tilde{\rho}}{1+\tilde{\rho}^{2}}\left(\frac{\partial \tilde{\rho}}{\partial x_{2}}\right)^{2}\right)\left[\frac{\lambda^{2}}{x_{1}^{2}}-\frac{\lambda^{2}}{d^{2}}\right] \frac{\partial \tilde{\rho}}{\partial y_{1}} \\
&+\gamma^{-2} \int_{\mathfrak{S}_{+}} \frac{\tilde{\rho}\left(\tilde{\rho}^{2}-1\right)}{\left(1+\rho^{2}\right)^{3}}\left(\left(\frac{\partial \varphi_{0}}{\partial x_{2}}\right)^{2}+2 \frac{\varphi_{0}}{\partial x_{2}} \frac{\varphi_{d}}{\partial x_{2}}+\left(\frac{\varphi_{d}}{\partial x_{2}}\right)^{2}\right)\left[\frac{\lambda^{2}}{x_{1}^{2}}-\frac{\lambda^{2}}{d^{2}}\right] \frac{\partial \tilde{\rho}}{\partial y_{1}} \\
&-\gamma^{-2} \int_{\mathfrak{S}_{+}} \frac{2 \tilde{\rho}\left(1-\tilde{\rho}^{2}\right)}{\left(1+\rho^{2}\right)^{3}}\left(\frac{\tilde{\rho}}{\partial x_{2}} \frac{\varphi_{0}}{\partial x_{2}}+\frac{\tilde{\rho}}{\partial x_{2}} \frac{\varphi_{s}}{\partial x_{2}}\right)\left[\frac{\lambda^{2}}{x_{1}^{2}}-\frac{\lambda^{2}}{d^{2}}\right] \frac{\partial \theta}{\partial y_{1}}+O(\epsilon) \\
&= O(\epsilon) .
\end{aligned}
$$

On the other hand, the last two terms in (7.2) can be estimated by

$$
\begin{aligned}
& \operatorname{Re}\left[\int_{\mathfrak{S}_{+}} \frac{1}{\left(1+\left|V_{d}\right|^{2}\right)^{2}} \overline{Z_{d}}\left(\Omega_{13}+\Omega_{23}\right) e^{i \tilde{\varphi}}\right] \\
& =\delta \gamma^{-1} \varepsilon|\log \varepsilon| \int_{\mathfrak{S}_{+}} \frac{1}{\left(1+|\tilde{\rho}|^{2}\right)^{2}}\left[\rho \frac{\partial \rho}{\partial x_{1}} \frac{\partial \varphi_{0}}{\partial x_{2}}-\rho \frac{\partial \rho}{\partial x_{2}} \frac{\partial \varphi_{0}}{\partial x_{1}}\right]+o(\varepsilon) \\
& =\delta \gamma^{-1} \varepsilon|\log \varepsilon| \int_{\mathbb{R}^{2}} \frac{\rho \rho^{\prime}}{|y|\left(1+\rho^{2}\right)^{2}} \mathrm{~d} y+o(\varepsilon) \\
& =\frac{\pi}{2} \delta \gamma^{-1} \varepsilon|\log \varepsilon|+o(\varepsilon) .
\end{aligned}
$$

As a conclusion, there holds

$$
\mathcal{C}_{\epsilon}(d)=c_{0}\left[\frac{\pi}{4 d \gamma^{2}} \log d-\frac{\pi}{2 \gamma} \delta \epsilon \log \frac{1}{\epsilon}+O(\epsilon)\right],
$$

where $c_{0} \neq 0$. Therefore, by recalling the parameters in (3.10), we obtain a solution to $\mathcal{C}_{\epsilon}(d)=0$ with the following asymptotic behavior:

$$
d_{\epsilon} \sim \frac{1+o(1)}{2 \epsilon}
$$

This proves Theorem 1.2.

Acknowledgment. J. Wei is partially supported by an Earmarked Grant from RGC of Hong Kong and a Focused Research Scheme of CUHK. J. Yang is supported by the foundations: NSFC(No.10901108), and FSZ(No. ZYC201006090108A, No. ZYC201105130099A). Part of this work was done when J. Yang visited the department of mathematics, the Chinese University of Hong Kong: he is very grateful to the institution for the kind hospitality. We thank Prof. T. C. Lin for useful discussions. We also thank the reviewer for the comments.

\section{REFERENCES}

[1] S. V. Alekseenko, P. A. Kuibin and V. L. Okulov, Theory of Concentrated Vortices: An Introduction, SpringerVerlag Berlin Heidelberg, 2007.

[2] C. Bardos, C. Sulem and P. Sulem, On the continuous limit for a system of classical spins, Comm. Math. Phys. 107 (1996), 431-454.

[3] F. Bethuel, H. Brezis and F. Hélein, Asymptotics for the minimization of a Ginzburg-Landau functional, Calc. Var. PDE. 1 (1993), no. 3, 123-148.

[4] F. Bethuel, H. Brezis and F. Hélein, Ginzburg-Landau vortices, Boston: Birkhäuser, 1994. 
[5] F. Bethuel, P. Gravejat and J. Saut, Existence and properties of travelling waves for the Gross-Pitaevskii equation, Stationary and time dependent Gross-Pitaevskii equations, 55-103, Contemp. Math., 473, Amer. Math. Soc., Providence, RI, 2008.

[6] F. Bethuel, P. Gravejat and J. Saut, Travelling waves for the Gross-Pitaevskii equation, II, Comm. Math. Phys. 285 (2009), no. 2, 567-651.

[7] F. Bethuel, G. Orlandi and D. Smets, Vortex rings for the Gross-Pitaevskii equation, J. Eur. Math. Soc. 6 (2004), no. 1, 17-94.

[8] F. Bethuel and J. C. Saut, Travelling waves for the Gross-Pitaevskii equation. I. Ann. Inst. H. Poincaré Phys. Theór. 70 (1999), no. 2, 147-238.

[9] N. H. Chang, J. Shatah and K. Uhlenbeck, Schrödinger maps, Comm. Pure Appl. Math. 53 (2000), no. 5, 590-602.

[10] D. Chiron, Travelling waves for the Gross-Pitaevskii equation in dimension larger than two, Nonlinear Anal. 58 (2004), no. 1-2, 175-204.

[11] D. Chiron, Vortex helices for the Gross-Pitaevskii equation, J. Math. Pures Appl. 84 (2005), no. 11, 1555-1647.

[12] J. Choe and J. Hoppe, Higher dimensional minimal submanifolds generalizing the catenoid and helicoid, Tohoku Math. J. 65 (2013), 43-55.

[13] M. del Pino, M. Kowalczyk and M. Musso, Variational reduction for Ginzburg-Landau vortices, J. Funct. Anal. 239 (2006), 497-541.

[14] M. del Pino, M. Musso and F. Pacard, Solutions of the Allen-Cahn equation which are invariant under screwmotion, Manuscripta Mathematica 138 (2012), no. 3-4, 273-286.

[15] S. Ding and B. Guo, Initial boundary value problem for the Landau-Lifshitz system (I)-Existence and partial regularity, Prog. Natural Sci. 8 (1998), no. 1, 11-23.

[16] S. Ding and B. Guo, Initial boundary value problem for the Landau-Lifshitz system (II)-Uniqueness, Prog. Natural Sci. 8 (1998), no. 2, 147-151.

[17] W. Y. Ding, On the Schrödinger flows, Proceedings of the International Congress of Mathematicians, Vol. II (Beijing, 2002), 283-291, Higher Ed. Press, Beijing, 2002.

[18] W. Y. Ding, H. Y. Tang and C. C. Zeng, Self-similar solutions of Schrödinger flows, Cal. Var. PDE 34 (2009), no. 2, 267-277.

[19] W. Y. Ding and Y. D. Wang, Schrödinger flow of maps into symplectic manifolds, Sci. China Ser. A 41 (1998), no. $7,746-755$.

[20] W. Y. Ding and Y. D. Wang, Local Schrödinger flow into Kähler manifolds, Sci. China Ser. A 44 (2001), no. $11,1446-1464$.

[21] B. Guo and M. Hong, The Landau-Lifschitz equation of the ferromagnetic spin chain and harmonic maps, Cal. Var. PDE 1 (1993), 311-334.

[22] S. Gustafson, K. Kang, T. P. Tsai, Asymptotic stability of harmonic maps under the Schrödinger flow, Duke Math. J. 145 (2008), no. 3, 537-583.

[23] S. Gustafson, K. Kang, T. P. Tsai, Schrödinger flow near harmonic maps, Comm. Pure Appl. Math. 60 (2007), no. $4,463-499$.

[24] S. Gustafson and J. Shatah, The stability of localized solutions of Landau-Lifshitz equations, Comm. Pure Appl. Math. 55 (2002), no. 9, 1136-1159.

[25] F. Hang and F. H. Lin, Static theory for planar ferromagnets an antiferromagnets, Acta Mathematica Sinica, English Series 17 (2001), 541-580.

[26] F. Hang and F. Lin, A Liouville type theorem for minimizing maps. Special issue dedicated to Daniel W. Stroock and Srinivasa S. R. Varadhan on the occasion of their 60th birthday, Methods Appl. Anal. 9 (2002), no. $3,407-424$.

[27] A. Hubert and R. Schafer, Magnetic Domain-The Analysis of Magnetic Microstructures, Berlin, Heidelberg, Springer-Verlag, 1998.

[28] J. Huhtamaki and P. Kuopanportti, Helical spin textures in dipolar Bose-Einstein condensates, Pysical Review A, 82 (2010), 053616.

[29] C. A. Jones and P. H. Roberts, Motion in a Bose condensate IV, Axisymmetric solitary waves, J. Phys. A 15 (1982), 2599-2619.

[30] S. Komineas and N. Papanicolaou, Topology and dynamics in ferromagnetic media. Phys. D. 99 (1996), no. 1, 81-107.

[31] S. Komineas and N. Papanicolaou, Vortex dynamics in two-dimensional antiferromagnets, Nonlinearity 11 (1998), no. 2, 265-290.

[32] L. Landau and E. Lifshitz, On the theory of the dispersion of magnetic permeability in ferromagnetic bodies, Phys. Z. Sow. 8 (1935), 153-169.

[33] F. H. Lin, J. Shatah, Soliton dynamics in planar ferromagnets and anti-ferromagnets, Journal of Zhejiang University Science 4 (2003), no. 5, 503-510.

[34] F. Lin and J. Wei, Travelling wave solutions of Schrödinger map equation, Comm. Pure Appl. Math. 63 (2010), no. $12,1585-1621$. 
[35] F. Lin and J. Xin, On the dynamical law of the Ginzburg-Landau vortices on the plane, Comm. Pure Appl. Math. 52 (1999), no. 10, 1189-1212.

[36] R. Moser, Partial regularity for Landau-Lifshitz equation, Preprint Series Max-Plancik-Institute for Mathematics in the Sciences 26/2002.

[37] J. C. Neu, Vortices in complex scalar fields, Phy. D 43 (1990), no. 2-3, 385-406.

[38] N. Papanicolaou and P. N. Spathis, Semitopological solitons in planar ferromagnets, Nonlinearity 12 (1999), no. 2, 285-302.

[39] J. Shatah, C. C. Zeng, Schrödinger maps and anti-ferromagnetic chains, Comm. Math. Phys. 262 (2006), no. 2, 299-315.

[40] C. Wang, On Landau-Lifshitz equations in dimensions at most four, Indiana Univ. Math. J. 55 (2006), 16151644 .

[41] J. D. Watson and F. H. C. Crick, Molecular structure of nucleic acids: a structure for deoxyribose nucleic acid, Nature 171 (1953), 737-738.

[42] A. Visintin, On Landau-Lifshitz equations for ferromagnetism, Japan J. Appl. Math. 2 (1985), 69-84.

[43] Y. Zhou and B. Guo, Existence of weak solution for boundary problems of systems of ferro-magnetic chain, Sci. Sinica Ser. A 27 (1984), no. 8, 799-811.

[44] Y. Zhou, B. Guo and S. Tan, Existence and uniqueness of smooth solutions for system of ferromagnetic chain, Sci. China, Ser. A 34 (1991), no. 3, 257-266.

J. Wei - Department of Mathematics, Chinese University of Hong Kong, Shatin, Hong Kong.

E-mail address: wei@math.cuhk.edu.hk

J. Yang - College of mathematics and computational sciences, Shenzhen University, Nanhai Ave 3688 , Shenzhen, China, 518060.

E-mail address: jyang@szu.edu.cn 